\title{
Reduction and reconstruction of stochastic differential equations via symmetries
}

\author{
Francesco C. De Vecchi*, Paola Morando ${ }^{\dagger}$ and Stefania Ugolini ${ }^{\ddagger}$
}

\begin{abstract}
An algorithmic method to exploit a general class of infinitesimal symmetries for reducing stochastic differential equations is presented and a natural definition of reconstruction, inspired by the classical reconstruction by quadratures, is proposed. As a side result the well-known solution formula for linear onedimensional stochastic differential equations is obtained within this symmetry approach. The complete procedure is applied to several examples with both theoretical and applied relevance.
\end{abstract}

Keywords: Stochastic Differential Equations, Symmetry

MSC numbers: $60 \mathrm{H} 10,58 \mathrm{D} 19$

\section{Introduction}

Stochastic differential equations (SDEs) and, more in general, stochastic processes provide a very useful tool for the description of many phenomena in physics, meteorology, biology, social sciences, economics or finance. For this reason there is a great interest in finding new simple and flexible models with some kind of analytical tractability. Just to mention some recent examples, in 12, 38 the Authors focus on models with closed form of the marginal probability density, while in 13 systems with explicit formulas for the moments are discussed. Moreover, in [11, 10, 15], systems with exact expression for the Fourier (or generalized Laplace) transform of some significant functionals of the process are considered.

These examples also suggest that the knowledge of closed-form expressions for some mathematical objects related with SDEs can be useful in order to formulate faster or more stable algorithms for numerical simulation (see e.g. 9, 23, 33]), to propose better estimators for statistical inference (see e.g. [5, 6, 16]) or to reduce the complexity of the models using asymptotic expansions or perturbation theory techniques (see e.g. [21, 32]).

For (deterministic) ordinary differential equations (ODEs) a powerful method to select general systems admitting closed analytical formulas for their solutions is the infinitesimal symmetries method originally proposed by Sophus Lie and thereafter developed by many Authors (see, e.g. 37, 42] and references therein). The underlying idea is to provide an algorithmic procedure in order to identify

\footnotetext{
${ }^{*}$ Dip. di Matematica, Università degli Studi di Milano, via Saldini 50, Milano, email: francesco.devecchi@unimi.it

${ }^{\dagger}$ DISAA, Università degli Studi di Milano, via Celoria 2, Milano, email: paola.morando@unimi.it

${ }^{\ddagger}$ Dip. di Matematica, Università degli Studi di Milano, via Saldini 50, Milano, email: stefania.ugolini@unimi.it
} 
symmetric ODEs and to exploit their symmetries for simplifying them.

Despite the well acknowledged and rich literature in the deterministic setting, the concept of infinitesimal symmetries for SDEs is quite recent [14, 18, 28, 30, 35, 45] and their use for reduction purposes is not yet completely developed.

The principal aim of this paper is to investigate the possible applications of a symmetry approach to SDEs taking the cue from the deterministic case. We propose a simple algorithmic method to exploit infinitesimal symmetries for reducing a SDE and, in the particular case of SDEs admitting a solvable Lie Algebra of symmetries, we provide a reconstruction procedure allowing to obtain the solution to the original SDE starting from the solution to the reduced one.

Although these basic applications of infinitesimal symmetries have already been discussed in [26, 28, 39, 45], there are several novelties in our approach. First of all we use the weaker notion of infinitesimal symmetry of a SDE proposed in 14], including both strong symmetries, used for reduction and reconstruction in [28], and quasi-strong symmetries used for reduction in [45]. It is worth to remark that our notion of symmetry allows us to take into account also random time change transformations which have not been considered by previous Authors. Moreover, the choice of a SDE formulation to deal with symmetries is more convenient than a formulation based on the generator operator of the SDE. Indeed, even though the notion of symmetry based on the generator is quite effective for reduction purposes, it turns out to be inadequate when one aims at generalizing to the stochastic framework the standard reconstruction by quadratures.

A further advantage of our method, inspired by the original ideas of Lie, is that we do not need the existence of a Lie group action related to the infinitesimal symmetries as required in [28, 45]. The possibility of working with both the global and the local action of a Lie group turns out to be very useful in order to deal with stochastically complete SDEs admitting infinitesimal symmetries which do not generate a globally defined flow of diffeomorphisms (see the example of Subsection 5.2).

Furthermore we propose a notion of reconstruction inspired by the classical idea of reconstruction by quadratures and similar to the one proposed in [26] for strong symmetries. We remark that this concept is different from the one proposed in 28] and our corresponding natural notion of integrability, which is in some respects more restrictive than that of [45] by not including higher order symmetries, allows us to exploit (not only Abelian but) general solvable algebras of symmetries. Besides, we recall that the stochastic quadrature procedure for one-dimensional diffusion processes proposed in [39] cannot be directly related to our results, since it is based on a well-defined variational structure. We discuss some particular examples of SDEs with a variational structure in Subsection 5.3

It is important to note that our approach is completely explicit and allows us to compute symmetries of a SDE by solving an overdetermined system of first order PDEs. In particular we apply our complete procedure to a class of onedimensional diffusions reducing to linear SDEs for a particular choice of the parameters. In this case, considering a suitable two-dimensional SDE including the original one, we are able to find the explicit solutions recovering the well known solution formula for one-dimensional linear SDEs, together with the usual change of variables coupled with the associated homogeneous equation.

Moreover we consider a class of (stochastic) mechanical models which includes the standard perturbations of stochastic Lagrangian systems. In particular, starting from a mechanical system describing a particle subjected to forces depending on the velocities, we look for general stochastic perturbations of the 
deterministic system preserving the symmetries and we analyze in details a couple of significant examples within this class.

Finally, we apply our symmetry approach to prove the integrability of a wellknown financial stochastic model (SABR) and we discuss the possible generalizations of our results suggested by this analysis.

The paper is organized as follows. In Section 2, for the convenience of the reader, we collect some general facts about foliations and reduction maps. In Section 3 we introduce the definition of infinitesimal symmetry of a SDE as proposed in [14] and we recall some results and formulas we need in the rest of the paper. Reduction, reconstruction and integrability of a SDE are described in Section 4 and in Section 5 the general theory is applied to some explicit relevant examples in order to point out the effectiveness of the proposed method.

\section{Some geometric preliminaries}

In this section we recall some geometric preliminaries needed in the following. In particular in Subsection 2.1 we introduce a class of foliations which turns out to be useful in order to reduce SDEs, whereas in Subsection 2.2 we describe adapted coordinate systems for solvable Lie algebras of vector fields which are exploited in the reconstruction process.

We work in an open set $M$ of the Euclidean space $\mathbb{R}^{n}$ with a cartesian coordinate system $x^{i}$ and we denote by $U\left(x_{0}\right)$ an open neighborhood of a point $x_{0} \in M$. All (real or matrix-valued) functions on $M$ are smooth and we denote by $\partial_{i}$ the partial derivatives with respect to $x^{i}$. If $A: M \rightarrow \operatorname{Mat}(k, m)$, we write $A_{r}^{l}$ for the $l$-th row and $r$-th column component of the matrix $A$, identifying $\operatorname{Mat}(k, 1)$ with $\mathbb{R}^{k}$.

Given a function $\Psi: M \rightarrow \mathbb{R}^{h}$ and a vector field $Y \in T M$, the push forward of $Y$ by $\Psi$ is defined by

$$
\Psi_{*}(Y)=\nabla(\Psi) \cdot Y
$$

where

$$
(\nabla(\Psi))_{j}^{i}=\partial_{j}\left(\Psi^{i}\right), \quad i=1, \ldots, h \quad j=1, \ldots, n
$$

and d denotes the usual matrix product. If $A: M \rightarrow \operatorname{Mat}(h, k)$ we define $Y(A): M \rightarrow \operatorname{Mat}(h, k)$ as

$$
(Y(A))_{j}^{i}=Y\left(A_{j}^{i}\right)
$$

where $Y\left(A_{j}^{i}\right)$ is the usual notation for the vector field $Y$ applied to the realvalued functions $A_{j}^{i}$.

In the following we denote by $I_{n}$ the $n$-dimensional identity matrix and by $S O(m)$ the group of the orthogonal matrices.

\subsection{Foliations and projections}

Let $Y_{1}, \ldots, Y_{k}$ be a set of vector fields on $M$ such that the distribution $\Delta=$ $\operatorname{span}\left\{Y_{1}, \ldots, Y_{k}\right\}$ is of constant rank $r$. If $\Delta$ is integrable, i.e. $\left[Y_{i}, Y_{j}\right] \in \Delta$ for every $i, j=1, \ldots, k$, then $\Delta$ defines a foliation on $M$. Moreover, if there is a submersion $\Psi: M \rightarrow M^{\prime}$, where (possibly restricting $M$ ) $M^{\prime}$ is an open subset of $\mathbb{R}^{n-r}$ such that

$$
\Delta=\operatorname{ker}(\nabla(\Psi))
$$

and the level sets of $\Psi$ are connected subsets of $M$, the foliation defined by $\Delta$ can be used for reduction purposes. In fact, under these assumptions, $\Psi$ is a surjective submersion and the level sets of $\Psi$ are connected closed submanifolds of $M$. 
Definition 2.1 A surjective submersion $\Psi: M \rightarrow M^{\prime}$ is a reduction map if $\Psi$ has connected level sets. The vector fields $Y_{1}, \ldots, Y_{k}$ generating an integrable distribution $\Delta$ of constant rank $r$ are reduction vector fields for the reduction $\operatorname{map} \Psi: M \rightarrow M^{\prime}$ if

$$
\operatorname{span}\left\{Y_{1}(x), \ldots, Y_{k}(x)\right\}=\operatorname{ker} \nabla(\Psi)(x) \quad \forall x \in M .
$$

We remark that, if $Y_{1}, \ldots, Y_{k}$ are reduction vector fields for the reduction map $\Psi$, then $\left(M, \Psi, M^{\prime}\right)$ is a fibred manifold.

Definition 2.2 A set of vector fields $Y_{1}, \ldots, Y_{r}$ on $M$ is regular on $M$ if, for any $x \in M$, the vectors $Y_{1}(x), \ldots, Y_{r}(x)$ are linearly independent.

A set of vector fields $Y_{1}, \ldots, Y_{k}$ generating an integrable distribution $\Delta$ of constant rank does not admit in general a global reduction map, but the following well known local result holds.

Proposition 2.3 (Frobenius theorem) Let $Y_{1}, \ldots, Y_{k}$ be a set of vector fields generating a regular integrable distribution $\Delta$ of constant rank $r$. Then, for any $x \in M$, there exist a neighborhood $U$ of $x$ and a reduction map $\Psi: U \rightarrow U^{\prime} \subset$ $\mathbb{R}^{n-r}$ such that $Y_{1}, \ldots, Y_{k}$ are reduction vector fields for $\Psi$.

We remark that the classical reduction of a manifold under a Lie group action is included in Definition 2.1. Indeed, given a connected Lie group $G$ acting on $M$, we can naturally define an equivalence relation and the quotient manifold $M^{\prime}=M / G:=M / \sim$. If the action of $G$ is proper and free, $M^{\prime}$ admits a natural structure of $(n-r)$-dimensional manifold (see [37]) and the natural projection $\Pi: M \rightarrow M^{\prime}$ is a submersion. Moreover, if $G$ is connected and $\left\{Y_{1}, \ldots, Y_{r}\right\}$ are the generators of the corresponding Lie algebra, $\Pi$ is a reduction map and

$$
\operatorname{span}\left\{Y_{1}(x), \ldots, Y_{r}(x)\right\}=\operatorname{ker}(\nabla(\Pi)(x)) \quad \forall x \in M .
$$

In this case $\left(M, \Psi, M^{\prime}\right)$ is not only a fibred manifold but also a principal bundle with structure group $G$.

Proposition 2.4 Let $\Psi: M \rightarrow M^{\prime}$ be a reduction map and suppose that the vector fields $\left\{Y_{1}, \ldots, Y_{k}\right\}$ are reduction vector fields for $\Psi$. If $M$ is connected, for any function $f \in C^{\infty}(M)$ such that $Y_{i}(f)=0$ there exists a unique function $f^{\prime} \in C^{\infty}\left(M^{\prime}\right)$ such that

$$
f=f^{\prime} \circ \Psi \text {. }
$$

Moreover, if $\mathcal{G}=\operatorname{span}\left\{Y_{1}, \ldots, Y_{k}\right\}$ and $Y$ is a vector field on $M$ such that

$$
[Y, \mathcal{G}] \subset \mathcal{G}
$$

there exists an unique vector field $Y^{\prime}$ such that

$$
\Psi_{*}(Y)=\nabla(\Psi) \cdot Y=Y^{\prime} \circ \Psi .
$$

Proof. See Chapter 4 of [36].

\subsection{Solvable algebras and adapted coordinate systems}

For later use, in this subsection we discuss the local existence of a suitable adapted coordinate system on $M$ such that the generators $Y_{1}, \ldots, Y_{r}$ of a solvable Lie algebra have a special form. 
Definition 2.5 Let $Y_{1}, \ldots, Y_{r}$ be a set of regular vector fields on $M$ which are generators of a solvable Lie algebra $\mathcal{G}$. We say that $Y_{1}, \ldots, Y_{r}$ are in canonical form if there are $i_{1}, \ldots, i_{l}$ such that $i_{1}+\ldots+i_{l}=r$ and

$$
\left(Y_{1}|\ldots| Y_{r}\right)=\left(\begin{array}{c|c|c|c}
I_{i_{1}} & G_{1}^{1}(x) & \ldots & G_{l}^{1}(x) \\
\hline 0 & I_{i_{2}} & \ldots & G_{l}^{2}(x) \\
\hline \vdots & \ddots & \ddots & \vdots \\
0 & 0 & \ldots & I_{i_{l}} \\
\hline 0 & 0 & 0 & 0
\end{array}\right),
$$

where $G_{k}^{h}: M \rightarrow \operatorname{Mat}\left(i_{h}, i_{k}\right)$ are smooth functions.

Theorem 2.6 Let $\mathcal{G}$ be an $r$-dimensional solvable Lie algebra on $M$ such that $\mathcal{G}$ has constant dimension $r$ as a distribution of $T M$ and let $\Psi$ be a reduction map for $\mathcal{G}$. Then, for any $x_{0} \in M$, there is a set of generators $Y_{1}, \ldots, Y_{r}$ of $\mathcal{G}$ and a local diffeomorphism $\Phi: U\left(x_{0}\right) \rightarrow \tilde{M}$ of the form

$$
\Phi=\left(\begin{array}{c}
\tilde{\Phi} \\
\Psi
\end{array}\right)
$$

such that $\Phi_{*}\left(Y_{1}\right), \ldots, \Phi_{*}\left(Y_{r}\right)$ are generators in canonical form for $\Phi_{*}(\mathcal{G})$.

Proof. Since $\mathcal{G}$ is solvable, denoting by $\mathcal{G}^{(0)}=\mathcal{G}$ and $\mathcal{G}^{(i+1)}=\left[\mathcal{G}^{(i)}, \mathcal{G}^{(i)}\right]$, there exists $l \geq 0$ such that $\mathcal{G}^{(l)} \neq 0$ and $\mathcal{G}^{(l+1)}=\{0\}$. Let $Y_{1}, \ldots, Y_{i_{1}}$ be the generators of $\mathcal{G}^{(l)}, \quad Y_{i_{1}+1}, \ldots, Y_{i_{1}+i_{2}}$ be the generators of $\mathcal{G}^{(l-1)} \backslash \mathcal{G}^{(l)}$ and, in general, $Y_{i_{1}+\ldots+i_{k-1}+1}, \ldots, Y_{i_{1}+\ldots+i_{k}}$ be the generators of $\mathcal{G}^{(l-k+1)} \backslash \mathcal{G}^{(l-k)}$. Since $\left(M, \Psi, M^{\prime}\right)$ is a fibred manifold, for any $x_{0} \in M$ we can consider a local smooth section $S: V\left(\Psi\left(x_{0}\right)\right) \rightarrow M$ defined in $V\left(\Psi\left(x_{0}\right)\right)$ and we can construct a local diffeomorphism on $W \times V$ (where $W \subset \mathbb{R}^{r}$ ) transporting $S\left(x_{0}\right)$ along the flows $\Phi_{a_{i}}^{i}$ of the vector fields $Y_{i}$. In particular, considering the function $F: W \times V \rightarrow M$ (where $W$ is a neighborhood of 0 in $\mathbb{R}^{r}$ ) defined by

$$
F\left(a_{1}, \ldots, a_{r}, x^{\prime 1}, \ldots x^{\prime n-r}\right)=\Phi_{a_{1}}^{1}\left(\ldots\left(\Phi_{a_{r}}^{r}\left(S\left(x^{1}, \ldots, x^{\prime r}\right)\right)\right) \ldots\right)
$$

we can define $\Phi=F^{-1}$. Indeed it is easy to prove that $F$ is a local diffeomorphism since $Y_{1}, \ldots, Y_{r}$ form a regular set of vector fields, $S$ is a local section of the foliation $\left(M, \Psi, M^{\prime}\right)$ and $\Psi$ is a reduction function for $Y_{1}, \ldots, Y_{r}$. Furthermore, since $F$ is obtained by composing the flows of $Y_{1}, \ldots, Y_{r}$ in the natural order (i.e. respecting the solvable structure of $\mathcal{G})$, it is easy to prove that $\Phi_{*}\left(Y_{1}\right), \ldots, \Phi_{*}\left(Y_{r}\right)$ are in canonical form.

Remark 2.7 In the particular case of a solvable connected Lie group G acting freely and regularly on $M$, Theorem [2.6 admits a global version. Indeed, under these hypotheses, $\Phi$ can be defined in an open set $U$ of the form $U=\Psi^{-1}(V)$ where $V$ is an open set of $M^{\prime}$ and $\left(M, \Psi, M^{\prime}\right)$ turns out to be a principal bundle with structure group $G$. So for a neighborhood $V$ of $\Psi\left(x_{0}\right)$ the set $U=\Psi^{-1}(V)$ is diffeomorphic to $V \times G$ and the generators $Y_{1}, \ldots, Y_{r}$ of $G$ are vertical vector fields with respect to the bundle structure of $M$. Furthermore, it is possible to choose a global coordinate system $g^{1}, \ldots, g^{r}$ on $G$ such that $Y_{1}, \ldots, Y_{r}$ are in canonical form (see for example [43] , Chapter 2, Section 3.1, Corollary 1) and equation (1) is given by $\Phi=\left(g^{1}, \ldots, g^{r}, \Psi^{1}, \ldots, \Psi^{n-r}\right)^{T}$. Obviously, if $\left(M, \Psi, M^{\prime}\right)$ is a trivial bundle, the diffeomorphism $\Phi$ of Theorem [2.6 can be defined globally. 


\section{Stochastic differential equations and their symmetries}

\subsection{Stochastic differential equations}

In the following, given a filtration $\mathcal{F}_{t} \subset \mathcal{F}$, we consider only stochastic processes $\left(\Omega, \mathcal{F}, \mathcal{F}_{t}, \mathbb{P}\right)$ which are adapted with the filtration $\mathcal{F}_{t}$.

Definition 3.1 Let $\mu: M \rightarrow \mathbb{R}^{n}$ and $\sigma: M \rightarrow \operatorname{Mat}(n, m)$ be two smooth functions. A stochastic process $X$ on $M$ and an $m$-dimensional Brownian motion $W$ (in short the process $(X, W)$ ) solve (in the weak sense) the SDE with coefficients $\mu, \sigma$ until the stopping time $\tau$ (or shortly solves the $S D E(\mu, \sigma)$ ) if for any $t \in \mathbb{R}_{+}$

$$
X_{t \wedge \tau}^{i}-X_{0}^{i}=\int_{0}^{t \wedge \tau} \mu^{i}\left(X_{s}\right) d s+\int_{0}^{t \wedge \tau} \sum_{\alpha=1}^{m} \sigma_{\alpha}^{i}\left(X_{s}\right) d W_{s}^{\alpha}
$$

If the process $(X, W)$ solves the $S D E(\mu, \sigma)$ we write, as usual,

$$
\begin{aligned}
d X_{t} & =\mu\left(X_{t}\right) d t+\sigma\left(X_{t}\right) \cdot d W_{t} \\
& =\mu d t+\sigma \cdot d W_{t} .
\end{aligned}
$$

The stopping time $\tau$ is supposed to be strictly less then the explosion time of the SDE.

It is well known that with a $\operatorname{SDE}(\mu, \sigma)$ it is possible to associate a second order differential operator

$$
L=\sum_{\alpha=1}^{m} \sum_{i, j=1}^{n} \frac{1}{2} \sigma_{\alpha}^{i} \sigma_{\alpha}^{j} \partial_{i j}+\sum_{i=1}^{n} \mu^{i} \partial_{i} .
$$

The operator $L$ is called the infinitesimal generator of the process and appears, for example, in the following important formula.

Proposition 3.2 (Ito formula) Let $(X, W)$ be a solution to the $S D E(\mu, \sigma)$ and let $f: M \rightarrow \mathbb{R}$ be a smooth function. Then $F=f(X)$ satisfies

$$
d F_{t}=L(f)\left(X_{t}\right) d t+\nabla(f)\left(X_{t}\right) \cdot \sigma\left(X_{t}\right) \cdot d W_{t} .
$$

\subsection{Stochastic transformations and symmetries of SDEs}

In this section we briefly recall the general definitions of finite and infinitesimal stochastic transformations of a SDE introduced in [14], in order to provide a general definition of symmetry, weak enough to include interesting examples in the stochastic framework.

Definition 3.3 Given a diffeomorphism $\Phi: M \rightarrow M^{\prime}$ and two smooth functions $B: M \rightarrow S O(m)$ and $\eta: M \rightarrow \mathbb{R}_{+}$, the triad $T=(\Phi, B, \eta)$ is called a stochastic transformation between $M$ and $M^{\prime}$. Stochastic transformations of the form $\left(\Phi, I_{m}, 1\right)$ are called strong whereas stochastic transformations of the form $(\Phi, B, 1)$ are called quasi-strong.

Hereafter we identify the strong stochastic transformations $T=\left(\Phi, I_{m}, 1\right)$ with the diffeomorphisms $\Phi$ and we denote by $S_{m}\left(M, M^{\prime}\right)$ the set of all stochastic transformations between $M$ and $M^{\prime}$.

A stochastic transformation $T=(\Phi, B, \eta)$ naturally defines two transformations 
on processes and SDEs. Indeed, if $H_{\eta}$ denotes the random time change given by the expression

$$
t^{\prime}=\int_{0}^{t} \eta\left(X_{s}\right) d s
$$

and $(X, W)$ is a process, we define $P_{T}(X, W)=\left(X^{\prime}, W^{\prime}\right)$ where

$$
\begin{gathered}
X^{\prime}=\Phi\left(H_{\eta}(X)\right) \\
W^{\prime}=H_{\eta}(\tilde{W}) .
\end{gathered}
$$

and

$$
\tilde{W}_{t}^{\alpha}=\int_{0}^{t} \sum_{\beta=1}^{m} B_{\beta}^{\alpha}\left(X_{s}\right) d W_{s}^{\beta} .
$$

Moreover, given a $\operatorname{SDE}(\mu, \sigma)$, we define $E_{T}(\mu, \sigma)=\left(\mu^{\prime}, \sigma^{\prime}\right)$ where

$$
\begin{aligned}
\mu^{\prime} & =\left(\frac{1}{\eta} L(\Phi)\right) \circ \Phi^{-1} \\
\sigma^{\prime} & =\left(\frac{1}{\sqrt{\eta}} \nabla(\Phi) \cdot \sigma \cdot B^{T}\right) \circ \Phi^{-1} .
\end{aligned}
$$

With these definitions it is easy to prove that if $(X, W)$ is a solution to $(\mu, \sigma)$, then $P_{T}(X, W)$ is a solution to $E_{T}(\mu, \sigma)$.

In [14] we prove that the set $S_{m}(M)=S_{m}(M, M)$ is a group with composition law defined by

$$
T^{\prime} \circ T=\left(\Phi^{\prime} \circ \Phi,\left(B^{\prime} \circ \Phi\right) \cdot B,\left(\eta^{\prime} \circ \Phi\right) \eta\right)
$$

and unit $1_{M}=\left(i d_{M}, I_{m}, 1\right)$. Moreover, if we consider the one-parameter group $T_{a}$ such that $T_{a} \circ T_{b}=T_{a+b}$ and $T_{0}=1_{M}$, there exist a vector field $Y$ and two smooth functions $C: M \rightarrow s o(m)$ and $\tau: M \rightarrow \mathbb{R}$ such that

$$
\begin{array}{rrr}
\partial_{a}\left(\Phi_{a}(x)\right) & = & Y\left(\Phi_{a}(x)\right) \\
\partial_{a}\left(B_{a}(x)\right) & = & C\left(\Phi_{a}(x)\right) \cdot B_{a}(x) \\
\partial_{a}\left(\eta_{a}(x)\right) & = & \tau\left(\Phi_{a}(x)\right) \eta_{a}(x) .
\end{array}
$$

Definition 3.4 The triad $V=(Y, C, \tau)$ defined by (2) is called infinitesimal stochastic transformation and the set of these triads is denoted by $V_{m}(M)$. The infinitesimal stochastic transformations of the form $(Y, 0,0)$ are called strong, whereas infinitesimal stochastic transformations of the form $(Y, C, 0)$ are called quasi-strong.

In the following we identify strong infinitesimal stochastic transformations $V=$ $(Y, 0,0)$ with the vector fields $Y$.

The set of infinitesimal stochastic transformations turns out to be a Lie algebra with respect to the Lie bracket

$$
\left[V_{1}, V_{2}\right]=\left(\left[Y_{1}, Y_{2}\right], Y_{1}\left(C_{2}\right)-Y_{2}\left(C_{1}\right)-\left\{C_{1}, C_{2}\right\}, Y_{1}\left(\tau_{2}\right)-Y_{2}\left(\tau_{1}\right)\right),
$$

where $\left\{C_{1}, C_{2}\right\}$ denotes the usual commutator between matrices. Moreover the action of a finite stochastic transformation on an infinitesimal one takes the form

$$
\begin{aligned}
T_{*}(V)= & \left((\nabla(\Phi) \cdot Y) \circ \Phi^{-1},\left(B \cdot C \cdot B^{-1}+Y(B) \cdot B^{-1}\right) \circ \Phi^{-1},\right. \\
& \left.\left(\tau+Y(\eta) \eta^{-1}\right) \circ \Phi^{-1}\right) .
\end{aligned}
$$

The important role of strong infinitesimal stochastic transformations in reduction of SDEs makes the following theorem very useful for applications. 
Theorem 3.5 Let $K=\operatorname{span}\left\{V_{1}, \ldots, V_{k}\right\}$ be a Lie algebra of infinitesimal stochastic transformations and $V_{i}=\left(Y_{i}, C_{i}, \tau_{i}\right)$. If $x_{0} \in M$ is such that $Y_{1}\left(x_{0}\right), \ldots, Y_{k}\left(x_{0}\right)$ are linearly independent, there exist an open neighborhood $U\left(x_{0}\right)$ and a stochastic transformation $T=\left(i d_{U}, B, \eta\right) \in S_{m}(U)$ such that $T_{*}\left(V_{1}\right), \ldots, T_{*}\left(V_{k}\right)$ are strong infinitesimal stochastic transformations. Furthermore the smooth functions $B, \eta$ are solutions to the equations

$$
\begin{aligned}
Y_{i}(B) & =-B \cdot C_{i} \\
Y_{i}(\eta) & =-\tau_{i} \eta
\end{aligned}
$$

for $i=1, \ldots, k$.

Proof. See [14].

In order to make the definition of a symmetry of a SDE more readable, we introduce the following notation: given $H \in T M$ and $K: M \rightarrow \operatorname{Mat}(n, r)$, we define the matrix-valued function $E:=[H, K]: M \rightarrow \operatorname{Mat}(n, r)$ by

$$
E_{j}^{i}=\sum_{k=1}^{n}\left(H^{k} \partial_{k}\left(K_{j}^{i}\right)-K_{j}^{k} \partial_{k}\left(H^{i}\right)\right) .
$$

It is easy to prove that the brackets $[\cdot, \cdot]$ generalize the standard Lie brackets for vector fields.

Definition 3.6 A stochastic transformation $T$ is a (finite) symmetry of a $S D E$ $(\mu, \sigma)$ if

$$
E_{T}(\mu, \sigma)=(\mu, \sigma)
$$

An infinitesimal stochastic transformation is an (infinitesimal) symmetry of the $\operatorname{SDE}(\mu, \sigma)$ if the following determining equations hold

$$
\begin{aligned}
{[Y, \sigma] } & =-\frac{1}{2} \tau \sigma-\sigma \cdot C \\
Y(\mu)-L(Y) & =-\tau \mu .
\end{aligned}
$$

It is easy to prove that $T \in S_{m}(M)$ is a symmetry of the $\operatorname{SDE}(\mu, \sigma)$ if and only if, for any solution $(X, W)$ to $(\mu, \sigma)$, also $P_{T}(X, W)$ is a solution to $(\mu, \sigma)$. Furthermore if $V \in V_{m}(M)$ generates a one parameter group $T_{a}, V$ is an (infinitesimal) symmetry of the $\operatorname{SDE}(\mu, \sigma)$ if and only if $T_{a}$ is a (finite) symmetry of $(\mu, \sigma)$. Moreover the following result holds.

Theorem 3.7 Let $V \in V_{m}(M)$ be a symmetry of the $S D E(\mu, \sigma)$. If $T \in$ $S_{m}(M)$, then $T_{*}(V)$ is a symmetry of $E_{T}(\mu, \sigma)$.

Corollary 3.8 Let $V_{1}=\left(Y_{1}, C_{1}, \tau_{1}\right), \ldots, V_{k}=\left(Y_{k}, C_{k}, \tau_{k}\right)$ be infinitesimal symmetries of $(\mu, \sigma)$. If $x_{0} \in M$ is such that $Y_{1}\left(x_{0}\right), \ldots, Y_{k}\left(x_{0}\right)$ are linearly independent, then there exist a neighborhood $U\left(x_{0}\right)$ and a stochastic transformation $T \in S_{m}\left(U, U^{\prime}\right)$ such that $T_{*}\left(V_{i}\right)$ are strong infinitesimal symmetries of $E_{T}(\mu, \sigma)$.

Remark 3.9 The concept of strong symmetry has been introduced, with the simple name of symmetry, in [28], where the noise is not necessarily a Brownian motion but any continuous semimartingale. It is easy to prove that the results of this section hold if we replace Brownian motion with any continuous semimartingale. Anyway in the following we restrict to Brownian motion since the weaker notion of symmetry in [14] has been introduced only for SDEs driven by Brownian motion. 


\section{Reduction and reconstruction}

In this section we propose a generalization of some well known results of symmetry reduction for ODEs to the stochastic framework. Moreover we provide suitable conditions for a symmetry to be inherited by the reduced equation and we tackle the problem of the reconstruction of the solution to the original SDE starting from the knowledge of the solution to the reduced one.

\subsection{Reduction}

Theorem 4.1 Let $\left\{Y_{1}, \ldots, Y_{k}\right\}$ be a set of reduction vector fields for the reduction map $\Psi: M \rightarrow M^{\prime}$ such that $\left(Y_{1}, C_{1}, 0\right), \ldots,\left(Y_{k}, C_{k}, 0\right)$ are quasi-strong symmetries of the $S D E(\mu, \sigma)$. If $\nabla(\Psi) \cdot \sigma \cdot C_{i}=0(\forall i=1, \ldots, k)$, there exists an unique $S D E\left(\mu^{\prime}, \sigma^{\prime}\right)$ on $M^{\prime}$ such that

$$
\begin{aligned}
L(\Psi) & =\mu^{\prime} \circ \Psi \\
\nabla(\Psi) \cdot \sigma & =\sigma^{\prime} \circ \Psi .
\end{aligned}
$$

Furthermore if $(X, W)$ is a solution to $(\mu, \sigma)$, then $(\Psi(X), W)$ is a solution to $\left(\mu^{\prime}, \sigma^{\prime}\right)$.

Before proving Theorem 4.1 we introduce the following lemma.

Lemma 4.2 If $(Y, C, \tau)$ is an infinitesimal symmetry of the $S D E(\mu, \sigma)$, for any smooth function $f$ we have

$$
\begin{aligned}
\nabla(Y(f)) \cdot \sigma-Y(\nabla(f) \cdot \sigma) & =\frac{1}{2} \tau \nabla(f) \cdot \sigma+\nabla(f) \cdot \sigma \cdot C . \\
L(Y(f))-Y(L(f)) & =\tau L(f) .
\end{aligned}
$$

Proof. See [14].

Proof of Theorem 4.1. Since we are considering quasi-strong symmetries, Lemma 4.2 ensures that $Y_{i}(L(\Psi))=L\left(Y_{i}(\Psi)\right)$ and, being $Y_{i} \in \operatorname{ker} \nabla(\Psi)$, we have $Y_{i}(L(\Psi))=0$. Hence Proposition 2.4 guarantees the existence of a function $\mu^{\prime}$ such that $L(\Psi)=\mu^{\prime} \circ \Psi$.

Moreover, in the case of quasi-strong symmetries, equation (5) reduces to $\left[Y_{i}, \sigma\right]=$ $-\sigma \cdot C_{i}$ and the hypothesis $\nabla(\Psi) \cdot \sigma \cdot C_{i}=0$ ensures that $\left[Y_{i}, \sigma\right] \in \operatorname{ker} \nabla(\Psi)$. Hence, denoting by $\sigma_{\alpha}$ the $\alpha$ column of $\sigma$, we have

$$
\left[Y_{i}, \sigma_{\alpha}\right] \in \operatorname{span}\left\{Y_{1}, \ldots, Y_{k}\right\} .
$$

Therefore, by Proposition 2.4 there exists an unique vector field $\sigma_{\alpha}^{\prime}$ on $M^{\prime}$ such that

$$
\sigma_{\alpha}^{\prime} \circ \Psi=\nabla(\Psi) \cdot \sigma_{\alpha}
$$

and, considering the matrix-valued function $\sigma^{\prime}$ with columns $\sigma_{\alpha}^{\prime}$, the theorem is proved.

Remark 4.3 If $Y_{1}, \ldots, Y_{k}$ are strong symmetries of the $S D E(\mu, \sigma)$, conditions $\nabla(\Psi) \cdot \sigma \cdot C_{i}=0$ of Theorem 4.1 are automatically satisfied. However in Section [5] we provide interesting examples of SDEs admitting only quasi-strong symmetries. Indeed a consequence of Theorem 2.10 in [45] is that if $V_{1}=$ $\left(Y_{1}, C_{1}, 0\right), \ldots, V_{k}=\left(Y_{k}, C_{k}, 0\right)$ are quasi-strong symmetries of $(\mu, \sigma)$ and $Y_{1}, \ldots, Y_{k}$ generate an integrable distribution of constant rank, there exists a (local) stochastic transformation $T=\left(i d_{M}, B, 1\right)$ such that $T_{*}\left(V_{1}\right), \ldots, T_{*}\left(V_{k}\right)$ satisfy the hypotheses of Theorem 4.1 for the transformed $S D E E_{T}(\mu, \sigma)$. We remark that, if the fibred manifold $\left(M, \Psi, M^{\prime}\right)$ is not a trivial fibred manifold, $T$ is only locally defined. 
Theorem 4.4 In the hypotheses and with the notations of Theorem 4.1, let $V=(Y, C, \tau)$ be a symmetry of the $\operatorname{SDE}(\mu, \sigma)$ such that, for any $i=1, \ldots, k$,

$$
\left[Y, Y_{i}\right] \in \operatorname{span}\left\{Y_{1}, \ldots, Y_{k}\right\}, \quad Y_{i}(C)=0, \quad Y_{i}(\tau)=0 .
$$

Then the infinitesimal transformation $\left(Y^{\prime}, C^{\prime}, \tau^{\prime}\right)$ on $M^{\prime}$, where $Y^{\prime}=\Psi_{*}(Y)$, $C^{\prime} \circ \Psi=C$ and $\tau^{\prime} \circ \Psi=\tau$, is a symmetry of the $S D E\left(\mu^{\prime}, \sigma^{\prime}\right)$.

Proof. We prove in detail that $Y^{\prime}$ satisfies the determining equation (5) for $\sigma^{\prime}$. The proof for $\mu^{\prime}$ can be easily obtained in a similar way.

Given $f \in C^{\infty}(M)$ such that $Y_{i}(f)=0$, Proposition 2.4 ensures that there exists a function $f^{\prime} \in C^{\infty}\left(M^{\prime}\right)$ such that $f=f^{\prime} \circ \Psi$. Moreover we have

$$
\begin{array}{ccc}
Y(f) & = & \left(Y^{\prime}\left(f^{\prime}\right)\right) \circ \Psi \\
\nabla(f) \cdot \sigma & = & \left(\nabla^{\prime}\left(f^{\prime}\right) \cdot \sigma^{\prime}\right) \circ \Psi,
\end{array}
$$

where $\nabla^{\prime}$ denotes the differential with respect to the coordinates $x^{i}$ of $M^{\prime}$. The determining equations (5) are equivalent to the relations

$$
Y^{\prime}\left(\sigma^{i}\right)-\nabla^{\prime}\left(Y^{\prime i}\right) \cdot \sigma^{\prime}=-\frac{1}{2} \tau^{\prime} \sigma^{i}-\sigma^{i} \cdot C^{\prime} .
$$

Since $(Y, C, \tau)$ is a symmetry of the $\operatorname{SDE}(\mu, \sigma)$, by Lemma 4.2 for any smooth function $f$ we have

$$
Y(\nabla(f) \cdot \sigma)-\nabla(Y(f)) \cdot \sigma=-\frac{1}{2} \tau \nabla(f) \cdot \sigma-\nabla(f) \cdot \sigma \cdot C .
$$

Applying equations (8) and (9) we obtain

$$
\begin{aligned}
\left\{Y^{\prime}\left(\sigma^{\prime i}\right)-\nabla^{\prime}\left(Y^{i}\right) \cdot \sigma^{\prime}\right\} \circ \Psi & =\left\{Y^{\prime}\left(\nabla^{\prime}\left(x^{i}\right) \cdot \sigma^{\prime}\right)-\nabla^{\prime}\left(Y^{\prime}\left(x^{i}\right)\right) \cdot \sigma^{\prime}\right\} \circ \Psi \\
& =Y\left(\left(\nabla^{\prime}\left(x^{i}\right) \cdot \sigma^{\prime}\right) \circ \Psi\right)-\nabla\left(Y^{\prime}\left(x^{i}\right) \circ \Psi\right) \cdot \sigma \\
& =Y\left(\nabla\left(\Psi^{i}\right) \cdot \sigma\right)-\nabla\left(Y\left(\Psi^{i}\right)\right) \cdot \sigma \\
& =-\frac{1}{2} \tau\left(\nabla\left(\Psi^{i}\right) \cdot \sigma\right)-\nabla\left(\Psi^{i}\right) \cdot \sigma \cdot C \\
& =\left(-\frac{1}{2} \tau^{\prime} \sigma^{\prime i}-\sigma^{\prime i} \cdot C^{\prime}\right) \circ \Psi .
\end{aligned}
$$

Since $\Psi$ is surjective, the thesis follows.

Remark 4.5 If $V_{1}=\left(Y_{1}, 0,0\right), \ldots, V_{k}=\left(Y_{k}, 0,0\right)$ are strong symmetries, conditions (7) of Theorem 4.4 on $V=(Y, C, \tau)$ can be rewritten as

$$
\left[V, V_{i}\right]=\sum_{j=1}^{k} \lambda_{j}^{i}(x) V_{j},
$$

where $\lambda_{i}^{j}(x)$ are smooth functions (in the particular case of $V_{1}, \ldots, V_{k}, V$ generating a finite dimensional Lie algebra, $\lambda_{j}^{i}$ are constants). In general, if $V_{i}$ are quasi-strong symmetries satisfying the hypotheses of Theorem 4.1. it is possible to prove an analogous of Theorem 4.4 using only hypotheses (10) but $C^{\prime}$ in the reduced symmetry $\left(Y^{\prime}, C^{\prime}, \tau^{\prime}\right)$ satisfies $C^{\prime} \circ \Psi=C+\tilde{C}$ where $\tilde{C}$ is such that $Y_{i}(C+\tilde{C})=0$ for $i=1, \ldots, k$ and $\nabla(\Psi) \cdot \sigma \cdot \tilde{C}=0$. 


\subsection{Reconstruction}

In this section we discuss the problem of reconstructing a process starting from the knowledge of the reduced one. In order to do this we need the following definition mainly inspired by ODEs framework.

Definition 4.6 Let $X$ and $Z$ be two processes on $M$ and $M^{\prime}$ respectively. We say that $X$ can be reconstructed from $Z$ until the stopping time $\tau$ if there exists a smooth function $F: \mathbb{R}^{k(m+1)} \times M^{\prime} \times M \rightarrow M$ such that

$X_{t}^{\tau}=F\left(\int_{0}^{t \wedge \tau} f_{0}\left(s, Z_{s}\right) d s, \int_{0}^{t \wedge \tau} f_{1}\left(s, Z_{s}\right) d W_{s}^{1}, \ldots, \int_{0}^{t \wedge \tau} f_{m}\left(s, Z_{s}\right) d W_{s}^{m}, Z_{t \wedge \tau}, X_{0}\right)$

where $W^{1}, \ldots, W^{m}$ are Brownian motions and $f_{i}: \mathbb{R} \times M^{\prime} \rightarrow \mathbb{R}^{k}$ are smooth functions. The process $X$ can be progressively reconstructed from $Z$ until the stopping time $\tau$ if there are some real processes $Z^{1}, \ldots, Z^{r}$, such that every $Z^{i}$ can be reconstructed until the stopping time $\tau$ starting from the process $\left(Z^{1}, \ldots, Z^{i-1}, Z\right)$, and $X$ can be reconstructed until the stopping time $\tau$ from the process $\left(Z^{1}, \ldots, Z^{r}, Z\right)$.

We remark that Definition 4.6 is general enough for our purposes (as we only consider Brownian motion driven SDEs), but can be easily generalized to include integration with respect more general stochastic processes.

Theorem 4.7 Let $\left\{Y_{1}, \ldots, Y_{r}\right\}$ be a set of regular reduction vector fields for the reduction map $\Psi: M \rightarrow M^{\prime}$ such that $Y_{1}, \ldots, Y_{r}$ generate a solvable algebra of strong symmetries for the $S D E(\mu, \sigma)$ and let $X^{x}$ be the unique solution to the $\operatorname{SDE}(\mu, \sigma)$ with Brownian motion $W$ such that $X_{0}^{x}=x$ almost surely. Then, for any $x \in M$, there exists a stopping time $\tau_{x}$ almost surely positive such that the process $X^{x}$ can be progressively reconstructed from $\Psi\left(X^{x}\right)$.

Proof. Let $\Phi$ be the diffeomorphism given by Theorem 2.6. defined in a neighborhood $U\left(x_{0}\right)$, and $T=\left(\Phi, I_{m}, 1\right)$. If $(\tilde{\mu}, \tilde{\sigma})=E_{T}(\mu, \sigma)$ then $\Phi_{*}\left(Y_{1}\right), \ldots, \Phi_{*}\left(Y_{r}\right)$ are symmetries of $(\tilde{\mu}, \tilde{\sigma})$ in canonical form and, denoting by $\tilde{x}^{i}$ the coordinate system on $\tilde{M}=\Phi(U)$, we have

$$
\frac{\partial}{\partial \tilde{x}_{i}}\left(\tilde{\mu}^{j}\right)=\frac{\partial}{\partial \tilde{x}_{i}}\left(\tilde{\sigma}_{\alpha}^{j}\right)=0
$$

for $i \leq r$ and $j \leq i$. This means that the $r$-th row of the $\operatorname{SDE}(\tilde{\mu}, \tilde{\sigma})$ does not depend on $\tilde{x}^{1}, \ldots, \tilde{x}^{r}$, the $(r-1)$-th row does not depend on $\tilde{x}^{1}, \ldots, \tilde{x}^{r-1}$ and so on. Hence the process $\tilde{X}=\Phi\left(X^{x_{0}}\right)$ can be progressively reconstructed from $\Pi(\tilde{X})$, where $\Pi$ is the projection of $\tilde{M}$ on the last $n-r$ coordinates. Since by definition of $\tilde{X}$ and $\Phi$ we have $\Pi(\tilde{X})=\Pi\left(\Phi\left(X^{x_{0}}\right)\right)=\Psi\left(X^{x_{0}}\right)$, the process $\tilde{X}$ can be progressively reconstructed from $\Psi\left(X^{x_{0}}\right)$. Moreover, being $X^{x_{0}}=\Phi^{-1}(\tilde{X})$ until the process $X^{x_{0}}$ exits from the open set $U$ we have that $\tilde{X}^{x_{0}}$ can be progressively reconstructed from $\Psi(X)$ until the stopping time

$$
\tau=\inf _{t \in \mathbb{R}_{+}}\left\{X_{t}^{x_{0}} \notin U\right\}
$$

that is almost surely positive since $U$ is a neighborhood of $x_{0}$.

Corollary 4.8 In the hypotheses and with the notations of Theorem 4.7 if the Lie algebra generated by $Y_{1}, \ldots, Y_{r}$ is Abelian then $X^{x}$ can be reconstructed from $\Psi\left(X^{x}\right)$.

Proof. If $\mathcal{G}=\operatorname{span}\left\{Y_{1}, \ldots, Y_{r}\right\}$ is Abelian, the diffeomorphism $\Phi$ of Theorem 2.6 rectifies $\mathcal{G}$. 
Remark 4.9 In order to compare our results with the reconstruction method proposed in [28] we consider the case of $Y_{1}, \ldots, Y_{r}$ generating a general Lie group $G$ whose action is free and proper. In this case $\left(M, \Psi, M^{\prime}\right)$ is a principal bundle with structure group $G$ and locally diffeomorphic to $U=V \times G$, where $V$ is an open subset of $\mathbb{R}^{n-r}$. If we denote by $\bar{X}$ the reduced process and by $g$ the coordinates on $G$ we have that the process $\bar{G}$ in $U$ satisfies the following Stratonovich equation

$$
d \bar{G}_{t}=\sum_{i=1}^{r} f_{0}^{i}\left(\bar{X}_{t}\right) Y_{i}\left(\bar{G}_{t}\right) d t+\sum_{\alpha=1}^{m} \sum_{i=1}^{r} f_{\alpha}^{i}\left(\bar{X}_{t}\right) Y_{i}\left(\bar{G}_{t}\right) \circ d W_{t}^{\alpha},
$$

where $f_{j}^{i}$ are smooth real-valued functions. Despite the fact that the knowledge of the reduced process $\bar{G}_{t}$ formally allows the reconstruction of the initial process $X_{t}$, for a general group $G$ this reconstruction cannot be reduced to quadratures. On the other hand, if $G$ is solvable, it is possible to choose a set of global coordinates on $G$ which reduce equation [11) to integration by quadratures as required by Definition 4.6 .

The following definition generalizes to the stochastic framework the well known definition of integrability for a system of ODEs.

Definition 4.10 A SDE $(\mu, \sigma)$ is completely integrable (or simply integrable) if for any $x \in M$ there exists an almost surely positive stopping time $\tau_{x}>0$ such that the solution process $X^{x}$ can be progressively reconstructed until the stopping time $\tau_{x}$ from a deterministic process.

Theorem 4.11 Let $(\mu, \sigma)$ be a SDE on $M \subset \mathbb{R}^{n}$ admitting an n-dimensional solvable Lie algebra $\mathcal{G}$ of strong symmetries which are also a regular set of vector fields. Then $(\mu, \sigma)$ is integrable.

Proof. Since $\mathcal{G}$ has the same dimension of $M$, the map $\Psi=0$, and the transformed $\operatorname{SDE}\left(\mu^{\prime}, \sigma^{\prime}\right)$ is such that the first row of $\mu^{\prime}$ and $\sigma^{\prime}$ does not depend on $x^{\prime 1}$, the second row does not depend on $x^{\prime 1}, x^{\prime 2}$ and so on. Therefore the $n$-th row of $\mu^{\prime}$ and $\sigma^{\prime}$ does not depend on any variables $x^{11}, \ldots, x^{\prime n}$ and so it is constant. This means that the solution $X^{\prime}=\Phi(X)$ can be progressively reconstructed from a constant process.

We remark that the hypotheses of Theorem 4.11 are only sufficient and not necessary. For example the SDE

$$
\left(\begin{array}{c}
d X_{t} \\
d Z_{t}
\end{array}\right)=\left(\begin{array}{c}
0 \\
g\left(X_{t}\right)
\end{array}\right)+\left(\begin{array}{c}
1 \\
f\left(X_{t}\right)
\end{array}\right) d W_{t}
$$

is obviously integrable, but it is easy to prove that (for general $g(x), f(x)$ ) it does not admit other symmetries than

$$
V=\left(\left(\begin{array}{l}
0 \\
1
\end{array}\right),\left(\begin{array}{ll}
0 & 0 \\
0 & 0
\end{array}\right), 0\right)
$$

\section{$5 \quad$ Examples}

In this section we apply our general reduction procedure to some explicit examples. Following the line of previous discussion, given a $\operatorname{SDE}(\mu, \sigma)$, we start by looking for a solvable algebra of symmetries $\mathcal{G}=\left\{V_{1}, \ldots, V_{r}\right\}$ for $(\mu, \sigma)$. Hence 
we compute a stochastic transformation $T=(\Phi, B, \eta)$ transforming $V_{1}, \ldots, V_{r}$ into strong symmetries $V_{k}^{\prime}=\left(Y_{k}^{\prime}, 0,0\right)$ for the transformed $\operatorname{SDE} E_{T}(\mu, \sigma)$ such that the vector fields $Y_{1}^{\prime}, \ldots, Y_{r}^{\prime}$ are in canonical form. Finally we use the results of Section 4 to reduce (or integrate) the transformed $\operatorname{SDE} E_{T}(\mu, \sigma)$ and we reconstruct the solution to $(\mu, \sigma)$ by means of the inverse transformation $T^{-1}$.

\subsection{A class of one-dimensional Kolmogorov-Pearson diffusions}

We consider the following class of SDE within the Kolmogorov-Pearson type diffusions

$$
d X_{t}=\left(\lambda X_{t}+\nu\right) d t+\sqrt{\alpha X_{t}^{2}+2 \beta X_{t}+\gamma} d W_{t}
$$

where $\alpha, \beta, \gamma, \lambda, \nu \in \mathbb{R}, \alpha \geq 0$ and $\alpha \gamma-\beta^{2} \geq 0$.

For $\alpha=\beta=0$ the class includes the Ornstein-Uhlenbeck process and for $\alpha \gamma-$ $\beta^{2}=0$ the important class of one-dimensional general linear SDEs of the form

$$
d X_{t}=\left(\lambda X_{t}+\nu\right)+\left(\sqrt{\alpha} X_{t}+\frac{\beta}{\sqrt{\alpha}}\right) d W_{t}
$$

Beyond the large number of applications of Ornstein-Uhlenbeck process and of linear SDEs and their spatial transformations, the Kolmogorov-Pearson class (12) has notable applications to finance (see [8, 41]), physics (see [17, 44]) and biology (see 20]). Moreover, there is a growing interest in the study of statistical inference (see 16]), in the analytical and spectral properties of the Kolmogorov equation associated with (12) (see [4]) and in the development of efficient numerical algorithms for its numerical simulation (see [9]). Finally the KolmogorovPearson diffusions are examples of "polynomial processes" that are becoming quite popular in financial mathematics (13]). For many particular values of the parameters $\alpha, \beta, \gamma, \lambda, \nu$ it is well known that equation (12) is an integrable SDE (first of all in the standard linear case corresponding to $\alpha \gamma-\beta^{2}=0$ ). Anyway this integrability property cannot be directly related to the existence of strong symmetries as showed by the following proposition.

Proposition 5.1 The $S D E\left(\lambda x+\nu, \sqrt{\alpha x^{2}+2 \beta x+\gamma}\right)$ admits strong symmetries if and only if

$$
\begin{aligned}
2 \beta \nu-2 \gamma \lambda+\alpha \gamma-\beta^{2} & =0 \\
\alpha \nu-\beta \lambda & =0 .
\end{aligned}
$$

Proof. The determining equations (5) for a strong symmetry $V=(Y, 0,0)$ of (12), with $Y=Y^{1} \partial_{x}$, are

$$
\begin{gathered}
\frac{(\alpha x+\beta)}{\sqrt{\alpha x^{2}+2 \beta x+\gamma}} Y^{1}-\partial_{x}\left(Y^{1}\right) \sqrt{\alpha x^{2}+2 \beta x+\gamma}=0 \\
\lambda Y^{1}-\frac{\left(\alpha x^{2}+2 \beta x+\gamma\right)}{2} \partial_{x x}\left(Y^{1}\right)-\partial_{x}\left(Y^{1}\right)(\lambda x+\nu)=0 .
\end{gathered}
$$

Equation (14) is an ODE in $Y^{1}$ with solution

$$
Y^{1}=Y_{0}^{1} \sqrt{\alpha x^{2}+2 \beta x+\gamma},
$$

where $Y_{0}^{1} \in \mathbb{R}$. Inserting the expression (16) in (15) we obtain

$$
Y_{0}^{1} \frac{\left(2 \beta \nu-2 \gamma \lambda+\alpha \gamma-\beta^{2}\right)+2 x(\alpha \nu-\beta \lambda)}{2 \sqrt{\alpha x^{2}+2 \beta x+\gamma}}=0
$$


and this concludes the proof.

We remark that a standard linear SDE of the form (13) admits a symmetry if and only if $\alpha \nu-\beta \lambda=0$. Therefore, in spite of their integrability, standard linear SDEs do not have, in general, strong symmetries.

In order to apply a symmetry approach to the study of the integrability of (12), we consider the following two-dimensional system:

$$
\left(\begin{array}{c}
d X_{t} \\
d Z_{t}
\end{array}\right)=\left(\begin{array}{c}
\lambda X_{t}+\nu \\
\lambda Z_{t}
\end{array}\right) d t+\left(\begin{array}{cc}
\sqrt{\alpha X_{t}^{2}+2 \beta X_{t}+\gamma} & 0 \\
\frac{Z_{t}\left(\alpha X_{t}+\beta\right)}{\sqrt{\alpha X_{t}^{2}+2 \beta X_{t}+\gamma}} & \frac{Z_{t} \sqrt{\alpha \gamma-\beta^{2}}}{\sqrt{\alpha X_{t}^{2}+2 \beta X_{t}+\gamma}}
\end{array}\right)\left(\begin{array}{c}
d W_{t}^{1} \\
d W_{t}^{2}
\end{array}\right),
$$

where $W_{t}^{1}:=W_{t}$. In the standard linear case, system (17) consists of SDE (13) and of the associated homogeneous one. If we look for the symmetries of system (17) of the form $V=(Y, C, \tau)$, where $Y=\left(Y^{1}, Y^{2}\right), C=\left(\begin{array}{cc}0 & c(x, z) \\ -c(x, z) & 0\end{array}\right)$ and $\tau(x, z)$, the determining equations are:

$$
\begin{gathered}
\frac{(\alpha x+\beta)}{\sqrt{\alpha x^{2}+2 \beta x+\gamma}} Y^{1}-\partial_{x}\left(Y^{1}\right) \sqrt{\alpha x^{2}+2 \beta x+\gamma}-\partial_{z}\left(Y^{1}\right) \frac{z(\alpha x+\beta)}{\sqrt{\alpha x^{2}+2 \beta x+\gamma}}+ \\
+\frac{\tau}{2} \sqrt{\alpha x^{2}+2 \beta x+\gamma}=0 \\
\frac{z\left(\alpha \gamma-\beta^{2}\right)}{\left(\sqrt{\alpha x^{2}+2 \beta x+\gamma}\right)^{3}} Y^{1}+\frac{\alpha x+\beta}{\sqrt{\alpha x^{2}+2 \beta x+\gamma}} Y^{2}-\partial_{x}\left(Y^{2}\right) \sqrt{\alpha x^{2}+2 \beta x+\gamma}+ \\
-\partial_{z}\left(Y^{2}\right) \frac{z(\alpha x+\beta)}{\sqrt{\alpha x^{2}+2 \beta x+\gamma}}+\frac{\tau}{2} \frac{z(\alpha x+\beta)}{\sqrt{\alpha x^{2}+2 \beta x+\gamma}}-\frac{c z \sqrt{\alpha \gamma-\beta^{2}}}{\sqrt{\alpha x^{2}+2 \beta x+\gamma}}=0 \\
-\frac{z \sqrt{\alpha \gamma-\beta^{2}}}{\sqrt{\alpha x^{2}+2 \beta x+\gamma}} \partial_{z}\left(Y^{1}\right)+c \sqrt{\alpha x^{2}+2 \beta x+\gamma}=0 \\
-\frac{z \sqrt{\alpha \gamma-\beta^{2}}(\alpha x+\beta)}{\left(\sqrt{\alpha x^{2}+2 \beta x+\gamma}\right)^{3}} Y^{1}+\frac{\sqrt{\alpha \gamma-\beta^{2}}}{\sqrt{\alpha x^{2}+2 \beta x+\gamma}} Y^{2}-\frac{z \sqrt{\alpha \gamma-\beta^{2}}}{\sqrt{\alpha x^{2}+2 \beta x+\gamma}} \partial_{z}\left(Y^{2}\right) \\
+c \frac{z(\alpha x+\beta)}{\sqrt{\alpha x^{2}+2 \beta x+\gamma}}+\frac{\tau}{2} \frac{z \sqrt{\alpha \gamma-\beta^{2}}}{\sqrt{\alpha x^{2}+2 \beta x+\gamma}}=0 \\
\lambda Y^{1}-(\lambda x+\nu) \partial_{x}\left(Y^{1}\right)-\lambda z \partial_{z}\left(Y^{1}\right)-\frac{\alpha x^{2}+2 \beta x+\gamma}{2} \partial_{x x}\left(Y^{1}\right)-\frac{\alpha z^{2}}{2} \partial_{z z}\left(Y^{1}\right)+ \\
-z(\alpha x+\beta) \partial_{x z}\left(Y^{1}\right)+\tau(\lambda x+\nu)=0 \\
\lambda Y^{2}-(\lambda x+\nu) \partial_{x}\left(Y^{2}\right)-\lambda z \partial_{z}\left(Y^{2}\right)-\frac{\alpha x^{2}+2 \beta x+\gamma}{2} \partial_{x x}\left(Y^{2}\right)-\frac{\alpha z^{2}}{2} \partial_{z z}\left(Y^{2}\right)+ \\
-z(\alpha x+\beta) \partial_{x z}\left(Y^{2}\right)+\tau \lambda z=0 .
\end{gathered}
$$

We can easily solve the previous overdetermined system of PDEs by a computer algebra software and we find two quasi-strong symmetries

$$
\begin{aligned}
& V_{1}=\left(Y_{1}, C_{1}, \tau_{1}\right)=\left(\left(\begin{array}{l}
z \\
0
\end{array}\right),\left(\begin{array}{cc}
0 & \frac{z \sqrt{\alpha \gamma-\beta^{2}}}{\alpha x^{2}+2 \beta x+\gamma} \\
-\frac{z \sqrt{\alpha \gamma-\beta^{2}}}{\alpha x^{2}+2 \beta x+\gamma} & 0
\end{array}\right), 0\right) \\
& V_{2}=\left(Y_{2}, C_{2}, \tau_{2}\right)=\left(\left(\begin{array}{l}
0 \\
z
\end{array}\right),\left(\begin{array}{ll}
0 & 0 \\
0 & 0
\end{array}\right), 0\right) .
\end{aligned}
$$

Therefore the function $\tilde{\Psi}: M \rightarrow \mathbb{R}$ given by $\tilde{\Psi}(x, z)=x$ is a reduction function with respect to the strong symmetry $Y_{2}$, being $\nabla(\tilde{\Psi}) \cdot Y_{2}=Y_{2}(\tilde{\Psi})=0$, and the reduced equation on $M^{\prime}=\tilde{\Psi}(M)=\mathbb{R}$ is exactly the original SDE (12).

This circumstance partially explains Proposition 5.1 since the original SDE (12) turns out as the reduction of the integrable system (17) with respect to the "wrong" symmetry, it does not inherit any symmetry.

In order to integrate system (17) and therefore also the original equation (12), we start by looking for a stochastic transformation $T=(\Phi, B, \eta)$ such that $T_{*}\left(V_{1}\right)$ 
and $T_{*}\left(V_{2}\right)$ are strong transformations and $\Phi_{*}\left(Y_{1}\right)$ and $\Phi_{*}\left(Y_{2}\right)$ are in canonical form. Since $V_{1}, V_{2}$ are quasi-strong infinitesimal stochastic transformations we can restrict to a quasi-strong transformation $T=(\Phi, B, 1)$.

Following the explicit construction of Theorem 2.6 the function $\Phi$ turns out to be both globally defined and globally invertible on $M$.

The vector fields $Y_{1}, Y_{2}$, whose flows are defined by

$$
\begin{aligned}
\Phi_{a^{1}}^{1}(x, z) & =\left(\begin{array}{c}
x+a^{1} z \\
z
\end{array}\right) \\
\Phi_{a^{2}}^{2}(x, z) & =\left(\begin{array}{c}
x \\
e^{a^{2}} z
\end{array}\right),
\end{aligned}
$$

generate a free and proper action of a solvable simply connected non Abelian Lie group on $M$. Hence, if we consider the point $p=(0,1)^{T}$ and the function $F: \mathbb{R}^{2} \rightarrow M$ given by

$$
F\left(a^{1}, a^{2}\right)=\Phi_{a^{1}}^{1}\left(\Phi_{a^{2}}^{2}(p)\right)=\left(\begin{array}{c}
a^{1} e^{a^{2}} \\
e^{a^{2}}
\end{array}\right),
$$

the function $\Phi: M \rightarrow \mathbb{R}^{2}$, which is the inverse of $F$, is given by

$$
\Phi(x, z)=\left(\begin{array}{c}
\frac{x}{z} \\
\log (z)
\end{array}\right) .
$$

By Theorem 3.5 the equations for $B$ are

$$
\begin{aligned}
& z \partial_{x}(B)=-B \cdot C_{1} \\
& z \partial_{z}(B)=0 .
\end{aligned}
$$

Writing:

$$
B=\left(\begin{array}{cc}
b & \sqrt{1-b^{2}} \\
-\sqrt{1-b^{2}} & b
\end{array}\right)
$$

from the second equation we deduce that $B$ does not depend on $z$ and from the first one we obtain that $b$ satisfies the equation

$$
\partial_{x} b=\frac{\sqrt{\alpha \gamma-\beta^{2}}}{\alpha x^{2}+2 \beta x+\gamma} \sqrt{1-b^{2}} .
$$

The latter equation is an ODE with separable variables admitting the following solution

$$
b=\frac{\alpha x+\beta}{\sqrt{\alpha} \sqrt{\alpha x^{2}+2 \beta x+\gamma}},
$$

and we get

$$
B=\left(\begin{array}{cc}
\frac{\alpha x+\beta}{\sqrt{\alpha} \sqrt{\alpha x^{2}+2 \beta x+\gamma}} & \frac{\sqrt{\alpha \gamma-\beta^{2}}}{\sqrt{\alpha} \sqrt{\alpha x^{2}+2 \beta x+\gamma}} \\
-\frac{\sqrt{\alpha \gamma-\beta^{2}}}{\sqrt{\alpha} \sqrt{\alpha x^{2}+2 \beta x+\gamma}} & \frac{\alpha x+\beta}{\sqrt{\alpha} \sqrt{\alpha x^{2}+2 \beta x+\gamma}}
\end{array}\right) .
$$

Putting $\left(x^{\prime}, z^{\prime}\right)^{T}=\Phi(x, z)$ we have

$$
\begin{aligned}
V_{1}^{\prime}=T_{*}\left(V_{1}\right) & =\left(\Phi_{*}\left(Y_{1}\right)=\left(\begin{array}{c}
1 \\
0
\end{array}\right),\left(\begin{array}{ll}
0 & 0 \\
0 & 0
\end{array}\right), 0\right), \\
V_{2}^{\prime}=T_{*}\left(V_{2}\right) & =\left(\Phi_{*}\left(Y_{2}\right)=\left(\begin{array}{c}
-x^{\prime} \\
1
\end{array}\right),\left(\begin{array}{ll}
0 & 0 \\
0 & 0
\end{array}\right), 0\right),
\end{aligned}
$$


and so $Y_{1}^{\prime}, Y_{2}^{\prime}$ are two generators of $\Phi_{*}(\mathcal{G})$ in canonical form. Introducing $d W_{t}^{\prime}=$ $B\left(X_{t}, Z_{t}\right) \cdot d W_{t}$ and applying Ito formula respectively to $\phi_{1}=x / z$ and $\phi_{2}=$ $\log (z)$ we can write equation (17) in the new variables:

$$
\begin{aligned}
d X_{t}^{\prime} & =(\nu-\beta) e^{-Z_{t}^{\prime}} d t+\frac{\beta e^{-Z_{t}^{\prime}}}{\sqrt{\alpha}} d W_{t}^{\prime 1}-\sqrt{\frac{\alpha \gamma-\beta^{2}}{\alpha}} e^{-Z_{t}^{\prime}} d W_{t}^{\prime 2} \\
d Z_{t}^{\prime} & =\left(\lambda-\frac{\alpha}{2}\right) d t+\sqrt{\alpha} d W_{t}^{\prime 1} .
\end{aligned}
$$

Since this is an integrable SDE, the solutions to equation (17) can be recovered following our general procedure. In particular, when $\alpha \gamma-\beta^{2}=0$, the solution to equation (12) is given by

$$
\begin{aligned}
& Z_{t}=Z_{0} e^{(\lambda-\alpha / 2) t+\sqrt{\alpha} W_{t}} \\
& X_{t}=Z_{t}\left(\frac{X_{0}}{Z_{0}}+\int_{0}^{t} \frac{\nu-\beta}{Z_{s}} d s+\int_{0}^{t} \frac{\beta}{\sqrt{\alpha} Z_{s}} d W_{s}\right)
\end{aligned}
$$

which is the well known explicit solution to the (general) linear one-dimensional SDE (13).

\subsection{Integrability of a singular SDE}

Let us consider the SDE on $M=\mathbb{R}^{2} \backslash\left\{(0,0)^{T}\right\}$

$$
\left(\begin{array}{c}
d X_{t} \\
d Z_{t}
\end{array}\right)=\left(\begin{array}{c}
\frac{\alpha X_{t}}{X_{t}^{2}+Z_{t}^{2}} \\
\frac{-\alpha Z_{t}}{X_{t}^{2}+Z_{t}^{2}}
\end{array}\right) d t+\left(\begin{array}{cc}
\frac{X_{t}^{2}-Z_{t}^{2}}{\sqrt{X_{t}^{2}+Z_{t}^{2}}} & 0 \\
0 & \frac{X_{t}^{2}-Z_{t}^{2}}{\sqrt{X_{t}^{2}+Z_{t}^{2}}}
\end{array}\right) \cdot\left(\begin{array}{c}
d W_{t}^{1} \\
d W_{t}^{2}
\end{array}\right)
$$

where $\alpha \in \mathbb{R}$. Despite the coefficients of $(\mu, \sigma)$ have a singularity in $(0,0)^{T}$, we will prove that the solution to (19) is not singular and that the explosion time of (19) is $+\infty$ for any deterministic initial condition $X_{0} \in M$.

A symmetry $V=(Y, C, \tau)$ of (19), with $Y=\left(Y^{1}, Y^{2}\right), C=\left(\begin{array}{cc}0 & c(x, z) \\ -c(x, z) & 0\end{array}\right)$ and $\tau(x, z)$, has to satisfy the following determining equations

$$
\begin{gathered}
\frac{x^{3}+3 x z^{2}}{\left(x^{2}+z^{2}\right)^{3 / 2}} Y^{1}-\frac{z^{3}+3 z x^{2}}{\left(x^{2}+z^{2}\right)^{3 / 2}} Y^{2}-\frac{x^{2}-z^{2}}{\sqrt{x^{2}+z^{2}}} \partial_{x}\left(Y^{1}\right)+\frac{1}{2} \tau \frac{x^{2}-z^{2}}{\sqrt{x^{2}+z^{2}}}=0 \\
-\frac{x^{2}-z^{2}}{\sqrt{x^{2}+z^{2}}} \partial_{x}\left(Y^{2}\right)-c \frac{x^{2}-z^{2}}{\sqrt{x^{2}+z^{2}}}=0 \\
-\frac{x^{2}-z^{2}}{\sqrt{x^{2}+z^{2}}} \partial_{z}\left(Y^{1}\right)+c \frac{x^{2}-z^{2}}{\sqrt{x^{2}+z^{2}}}=0 \\
\frac{x^{3}+3 x z^{2}}{\left(x^{2}+z^{2}\right)^{3 / 2}} Y^{1}-\frac{z^{3}+3 z x^{2}}{\left(x^{2}+z^{2}\right)^{3 / 2}} Y^{2}-\frac{x^{2}-z^{2}}{\sqrt{x^{2}+z^{2}}} \partial_{z}\left(Y^{2}\right)+\frac{1}{2} \tau \frac{x^{2}-z^{2}}{\sqrt{x^{2}+z^{2}}}=0 \\
-\frac{\alpha\left(x^{2}-z^{2}\right)}{\left(x^{2}+z^{2}\right)^{2}} Y^{1}-\frac{2 \alpha x z}{\left(x^{2}+z^{2}\right)^{2}} Y^{2}-\frac{\alpha x}{x^{2}+z^{2}} \partial_{x}\left(Y^{1}\right) \\
+\frac{\alpha z}{x^{2}+z^{2}} \partial_{z}\left(Y^{1}\right)-\frac{1}{2} \frac{\left(x^{2}-z^{2}\right)^{2}}{x^{2}+z^{2}}\left(\partial_{x x}\left(Y^{1}\right)+\partial_{z z}\left(Y^{1}\right)\right)+\tau \frac{\alpha x}{x^{2}+z^{2}}=0 \\
\frac{2 \alpha x z}{\left(x^{2}+z^{2}\right)^{2}} Y^{1}-\frac{\alpha\left(x^{2}-z^{2}\right)}{\left(x^{2}+z^{2}\right)^{2}} Y^{2}-\frac{\alpha x}{x^{2}+z^{2}} \partial_{x}\left(Y^{2}\right) \\
+\frac{\alpha z}{x^{2}+z^{2}} \partial_{z}\left(Y^{2}\right)-\frac{1}{2} \frac{\left(x^{2}-z^{2}\right)^{2}}{x^{2}+z^{2}}\left(\partial_{x x}\left(Y^{2}\right)+\partial_{z z}\left(Y^{2}\right)\right)-\tau \frac{\alpha z}{x^{2}+z^{2}}=0
\end{gathered}
$$


Solving this system of PDEs by a computer algebra software we find the unique (quasi-strong) symmetry

$$
V=(Y, C, \tau)=\left(\left(\begin{array}{c}
\frac{z}{x^{2}+z^{2}} \\
\frac{x}{x^{2}+z^{2}}
\end{array}\right),\left(\begin{array}{cc}
0 & \frac{x^{2}-z^{2}}{\left(x^{2}+z^{2}\right)^{2}} \\
-\frac{x^{2}-z^{2}}{\left(x^{2}+z^{2}\right)^{2}} & 0
\end{array}\right), 0\right)
$$

which unfortunately does not generate a one parameter group of stochastic transformations, as the trajectories of the points of the form $(h, h)$ and $(h,-h)$ (with $h \in \mathbb{R} \backslash\{0\})$, reach $(0,0)$ in a finite time.

Hence, in order to find a stochastic transformation $T=(\Phi, B, \eta)$ such that $T_{*}(V)$ is a strong transformation and $\Phi_{*}(Y)$ is in canonical form we have to solve the equations $Y(\Phi)=(1,0)^{T}$ and $Y(B)=-B \cdot C$. Writing $\Phi=(\tilde{\Phi}, \Psi)^{T}$, the map $\Psi$ has to solve $Y(\Psi)=0$, i.e.

$$
\frac{z}{x^{2}+z^{2}} \partial_{x}(\Psi)+\frac{x}{x^{2}+z^{2}} \partial_{z}(\Psi)=0 .
$$

By using the method of the characteristics, we immediately obtain a particular solution

$$
\Psi(x, z)=x^{2}-z^{2} .
$$

In order to find an adapted coordinate system for the Abelian Lie algebra $\mathcal{G}=$ $\operatorname{span}\{Y\}$ we have to solve the equation $Y(\tilde{\Phi})=1$, i.e.

$$
z \partial_{x}(\tilde{\Phi})+x \partial_{z}(\tilde{\Phi})=x^{2}+z^{2}
$$

Once again, applying the method of the characteristics, we obtain

$$
\tilde{\Phi}(x, z)=x z,
$$

so that we can consider the local diffeomorphism of $M=\mathbb{R}^{2} \backslash\left\{(0,0)^{T}\right\}$

$$
\Phi(x, z)=\left(\begin{array}{c}
\tilde{\Phi} \\
\Psi
\end{array}\right)=\left(\begin{array}{c}
x z \\
x^{2}-z^{2}
\end{array}\right)
$$

which is only locally invertible.

To construct the matrix-valued function $B$ of the form (18) we solve the equation $Y(B)=-B \cdot C$. In the new coordinates $\left(x^{\prime}, z^{\prime}\right)=\Phi(x, z)$ the equation becomes

$$
\partial_{x^{\prime}} b=\frac{z^{\prime}}{4 x^{\prime 2}+z^{\prime 2}} \sqrt{1-b^{2}}
$$

whose solution is

$$
b= \pm \frac{\sqrt{\sqrt{z^{\prime 2}+4 x^{\prime 2}}-z^{\prime}}}{\sqrt{2}\left(z^{\prime 2}+4 x^{\prime 2}\right)^{1 / 4}}
$$

and, coming back to the original coordinate system, we find

$$
B=\left(\begin{array}{cc}
\frac{z}{\sqrt{x^{2}+z^{2}}} & \frac{x}{\sqrt{x^{2}+z^{2}}} \\
\frac{-x}{\sqrt{x^{2}+z^{2}}} & \frac{z}{\sqrt{x^{2}+z^{2}}}
\end{array}\right)
$$

The transformed $\operatorname{SDE}\left(\mu^{\prime}, \sigma^{\prime}\right)=E_{T}(\mu, \sigma)$ has coefficients

$$
\begin{aligned}
\mu^{\prime} & =L(\Phi) \circ \Phi^{-1}=\left(\begin{array}{c}
0 \\
2 \alpha
\end{array}\right) \\
\sigma^{\prime} & =(\nabla(\Phi) \cdot \sigma) \circ \Phi^{-1}=\left(\begin{array}{cc}
z^{\prime} & 0 \\
0 & -2 z^{\prime}
\end{array}\right)
\end{aligned}
$$


and, by applying Ito formula, the original two-dimensional SDE becomes

$$
\begin{aligned}
d X_{t}^{\prime} & =Z_{t}^{\prime} d W_{t}^{\prime 1} \\
d Z_{t}^{\prime} & =2 \alpha d t-2 Z_{t}^{\prime} d W_{t}^{\prime 2},
\end{aligned}
$$

where $d W_{t}^{\prime}=B\left(X_{t}, Z_{t}\right) \cdot d W_{t}$. Since the equation in $Z^{\prime}$ is linear, the above SDE is integrable and therefore also (19) is integrable. Furthermore, since the map $\Phi: M \rightarrow \mathbb{R}^{2} \backslash\left\{(0,0)^{T}\right\}$ is a double covering map and since the SDE (20) has explosion time $\tau=+\infty$, the SDE (19), although singular at the origin, has also explosion time $\tau=+\infty$ for any deterministic initial condition $X_{0} \in M$.

This example point out the importance of developing a local reduction theory for SDEs, since in this case a global approach cannot be successful.

\subsection{Stochastic perturbation of mechanical equations}

In this example we analyze a wide class of models, related to (stochastic) mechanics, of the form

$$
\begin{aligned}
& d X_{t}^{i}=V_{t}^{i} d t \\
& d V_{t}^{i}=F_{0}^{i}\left(X_{t}, V_{t}\right) d t+\sum_{\alpha} F_{\alpha}^{i}\left(X_{t}\right) d W_{t}^{\alpha},
\end{aligned}
$$

i.e. with SDE coefficients:

$$
\begin{aligned}
\mu & =\left(\begin{array}{c}
v^{1} \\
\vdots \\
v^{n} \\
F_{0}^{1}(x, v) \\
\vdots \\
F_{0}^{n}(x, v)
\end{array}\right) \\
\sigma= & \left(\begin{array}{ccc}
0 & \cdots & 0 \\
\vdots & \vdots & \vdots \\
F_{1}^{1}(x) & \cdots & F_{m}^{1}(x) \\
\vdots & \vdots & \vdots \\
F_{1}^{n}(x) & \cdots & F_{m}^{n}(x)
\end{array}\right),
\end{aligned}
$$

where $\left(x^{i}, v^{i}\right)$ is the standard coordinate system of $M=\tilde{M} \times \mathbb{R}^{n}$ and $\tilde{M}$ is an open set of $\mathbb{R}^{n}$.

This kind of SDEs, representing a stochastic perturbation of the Newton equations for $n$ particles of mass $m_{i}=1$ subjected to forces depending on the positions and on the velocities

$$
\frac{d^{2} X_{t}^{i}}{d t^{2}}=F^{i}\left(X_{t}, \frac{d X_{t}}{d t}\right)
$$

arise in many contexts of mathematical physics. The class includes the Langevin type equation often used in the framework of Stochastic Thermodynamics (see, e.g., 34, 40]) for $F_{\alpha}^{i}=\delta_{\alpha}^{i}$ and $F_{0}^{i}=-\gamma V^{i}+\partial_{i}(U)(x)$, where $U: \mathbb{R}^{n} \rightarrow \mathbb{R}$ is a smooth function and $\gamma \in \mathbb{R}_{+}$. Furthermore, if the forces $F_{0}^{i}$ arise from a Lagrangian $L$ of the form

$$
L=\frac{1}{2} \sum_{i, j} g_{i, j}(x) v^{i} v^{j}-U(x)
$$


(where $g_{i, j}(x)$ is a metric tensor on $\mathbb{R}^{n}$ ) and the random perturbations $F_{\alpha}^{i}$ are given by

$$
F_{\alpha}^{i}=\sum_{j} g^{i, j}(x) \partial_{j}\left(U_{\alpha}\right)
$$

with $U_{\alpha}$ smooth functions, (21) turns out to be a Lagrangian system with the following action functional

$$
S=\int_{0}^{t} L\left(X_{s}, V_{s}\right) d s+\sum_{\alpha} \int_{0}^{t} U_{\alpha}\left(X_{s}\right) d W_{s}^{\alpha}
$$

(see, e.g., 7, 27]). There is a growing interest for this kind of stochastic perturbations of Lagrangian and Hamiltonian systems due both to their special mathematical properties and to their applications in mathematical physics (see, e.g., [2, 3, 24, 25, 30, 31, 39]).

In the following we propose a method to obtain a SDE of the form (21) which can be interpreted as a symmetric stochastic perturbation of a symmetric ODE of the form (22).

Given a vector field $\tilde{Y}_{0}=\left(\tilde{Y}_{0}^{1}(x), \ldots, \tilde{Y}_{0}^{n}(x)\right)^{T}$ on $\tilde{M}$ which is a symmetry of (22), the vector field

$$
Y=\left(\begin{array}{c}
\tilde{Y}_{0}^{1}(x) \\
\vdots \\
\tilde{Y}_{0}^{n}(x) \\
\sum_{k=1}^{n} \partial_{x^{k}}\left(\tilde{Y}_{0}^{1}\right) v^{k} \\
\vdots \\
\sum_{k=1}^{n} \partial_{x^{k}}\left(\tilde{Y}_{0}^{n}\right) v^{k}
\end{array}\right),
$$

is a symmetry of (21), when $F_{\alpha}^{i}=0$.

If $\tilde{Y}_{\alpha}=\left(\tilde{Y}_{\alpha}^{1}(x), \ldots, \tilde{Y}_{\alpha}^{n}(x)\right)$, for $\alpha=1, \ldots, m$, are $m$ vector fields on $\tilde{M}$ such that there exists a matrix-valued function $C: \mathbb{R}^{n} \rightarrow s o(m)$ satisfying

$$
\left[\tilde{Y}_{0}, \tilde{Y}_{\alpha}\right]=-\sum_{\beta=1}^{m} C_{\alpha}^{\beta}(x) \tilde{Y}_{\beta}
$$

and we set

$$
F_{\alpha}^{i}(x)=\tilde{Y}_{\alpha}^{i}(x), \quad i=1, \ldots, n
$$

we find that $V=(Y, C, 0)$ is a quasi-strong symmetry for the system (21). Indeed, the first determining equation (5) for $i=n+1, \ldots, 2 n$ becomes:

$$
\begin{aligned}
{[Y, \sigma]_{\alpha}^{i} } & =\sum_{j=1}^{2 n}\left(Y^{j} \partial_{j}\left(\sigma_{\alpha}^{i}\right)-\sigma_{\alpha}^{j} \partial_{j}\left(Y^{i}\right)\right) \\
& =\sum_{j=1}^{n} \tilde{Y}_{0}^{j} \partial_{x^{j}}\left(\tilde{Y}_{\alpha}^{i-n}\right)-\sum_{j=n+1}^{2 n} \tilde{Y}_{\alpha}^{j-n} \partial_{v^{j}}\left(\sum_{k=1}^{n} \partial_{x^{k}}\left(\tilde{Y}_{0}^{i}\right) v^{k}\right) \\
& =\sum_{j=1}^{n} \tilde{Y}_{0}^{j} \partial_{x^{j}}\left(\tilde{Y}_{\alpha}^{i-n}\right)-\sum_{j=1}^{n} \tilde{Y}_{\alpha}^{j} \partial_{x^{j}}\left(\tilde{Y}_{0}^{i-n}\right) \\
& =\left[\tilde{Y}, \tilde{Y}_{\alpha}\right]^{i-n}=-\sum_{\beta=1}^{m} C_{\alpha}^{\beta} \tilde{Y}_{\beta}^{i}=-\sum_{\beta=1}^{m} C_{\alpha}^{\beta} \sigma_{\beta}^{i} .
\end{aligned}
$$

Since $\sigma_{\alpha}^{i}=0$ for $i \leq n$ and $Y^{i}$ does not depend on $v$ for $i \leq n$, we have

$$
[Y, \sigma]_{\alpha}^{i}=-\sum_{\beta=1}^{m} C_{\alpha}^{\beta} \sigma_{\beta}^{i}, \quad i \leq n .
$$


Furthermore

$$
Y\left(\mu^{i}\right)-L\left(Y^{i}\right)=Y\left(\mu^{i}\right)-\mu\left(Y^{i}\right)=[Y, \mu]^{i}=0
$$

because $Y$ is a symmetry of (22).

An interesting particular case within this class is given by the following equation

$$
\frac{d^{2} X_{t}}{d t}=-\gamma \frac{d X_{t}}{d t}
$$

representing (for $\gamma>0$ ) the motion of a particle subjected to a linear dissipative force. This equation has the symmetry $\tilde{Y}=x \partial_{x}$, so that the system

$$
\left(\begin{array}{c}
d X_{t} \\
d V_{t}
\end{array}\right)=\left(\begin{array}{c}
V_{t} \\
-\gamma V_{t}
\end{array}\right) d t+\left(\begin{array}{c}
0 \\
\alpha X_{t}
\end{array}\right) d W_{t},
$$

which provides the equation of a dissipative random harmonic oscillator, has the strong symmetry

$$
Y=\left(\begin{array}{l}
x \\
v
\end{array}\right)
$$

If we consider the local diffeomorphism

$$
\Phi(x, v)=\left(\begin{array}{c}
\frac{1}{2} \log \left(x^{2}+v^{2}\right) \\
\frac{v}{x}
\end{array}\right),
$$

in the coordinates $\left(x^{\prime}, v^{\prime}\right)^{T}=\Phi(x, v)$ equation (23) becomes

$$
\begin{aligned}
d X_{t}^{\prime} & =\left(-\frac{\gamma\left(V_{t}^{\prime}\right)^{2}-V_{t}^{\prime}}{1+V_{t}^{\prime 2}}+\alpha^{2} \frac{1-V_{t}^{\prime 2}}{2+4 V_{t}^{\prime 2}+2 V_{t}^{\prime 4}}\right) d t+\frac{\alpha V_{t}^{\prime}}{1+V_{t}^{\prime 2}} d W_{t} \\
d V_{t}^{\prime} & =\left(-\gamma V_{t}^{\prime}-V_{t}^{\prime 2}\right) d t+\alpha d W_{t} .
\end{aligned}
$$

This system is not integrable but the equation for $V_{t}^{\prime}$ is known in literature (see [19]). Furthermore, as well as its deterministic counterpart, this equation admits a superposition rule 29].

Another interesting example within the class of the equations described by (21) is given by the following system

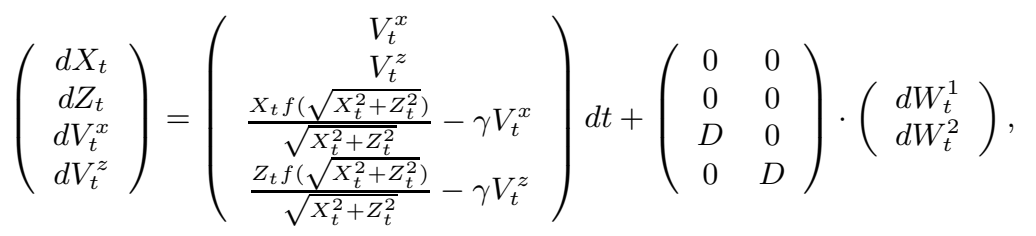

where $D \in \mathbb{R}_{+}$and $f: \mathbb{R}_{+} \rightarrow \mathbb{R}$ is a smooth function. The SDE (24) is a Langevin type equation describing a point particle of unitary mass subjected to the central force $f\left(\sqrt{x^{2}+z^{2}}\right)$, to an isotropic dissipation linear in the velocities and to a space homogeneous random force. Since both the central force and the dissipation are invariant under the rotation group, the vector field

$$
\tilde{Y}=\left(\begin{array}{c}
z \\
-x
\end{array}\right)
$$

is a symmetry of (24) for $D=0$. Furthermore we have that

$$
\left[\tilde{Y},\left(\begin{array}{ll}
1 & 0 \\
0 & 1
\end{array}\right)\right]=-\left(\begin{array}{ll}
1 & 0 \\
0 & 1
\end{array}\right) \cdot\left(\begin{array}{cc}
0 & 1 \\
-1 & 0
\end{array}\right) .
$$


So putting

$$
\begin{aligned}
Y & =\left(\begin{array}{c}
z \\
-x \\
v^{z} \\
-v^{x}
\end{array}\right) \\
C & =\left(\begin{array}{cc}
0 & 1 \\
-1 & 0
\end{array}\right)
\end{aligned}
$$

the infinitesimal stochastic transformation $V=(Y, C, 0)$ is a quasi-strong symmetry for equation (24).

In order to reduce (24) using the symmetry $V$, we have to find the stochastic transformation $T=(\Phi, B, 1)$ which puts $V$ in canonical form. Solving the equations for $\Phi$ and $B$ we obtain

$$
\begin{aligned}
& \Phi\left(x, z, v^{x}, v^{z}\right)=\left(\begin{array}{c}
\operatorname{acos}\left(\frac{x}{\sqrt{x^{2}+z^{2}}}\right) \\
\sqrt{z^{2}+x^{2}} \\
\frac{x v^{z}-z v^{x}}{x^{2}+z^{2}} \\
\frac{x v^{2}+z v^{2}}{\sqrt{x^{2}+z^{2}}}
\end{array}\right) \\
& B\left(x, z, v^{x}, v^{z}\right)=\left(\begin{array}{cc}
\frac{x}{\sqrt{x^{2}+x^{2}}} & \frac{z}{\sqrt{x^{2}+z^{2}}} \\
-\frac{z}{\sqrt{x^{2}+z^{2}}} & \frac{x}{\sqrt{x^{2}+z^{2}}}
\end{array}\right) .
\end{aligned}
$$

With the new coordinates $\Phi=\left(\theta, r, v^{\theta}, v^{r}\right)^{T}$ and with the new Brownian motion $d W_{t}^{\prime}=B \cdot d W_{t}$ we have

$$
\begin{aligned}
d \Theta_{t} & =V_{t}^{\theta} d t \\
d R_{t} & =V_{t}^{r} d t \\
d V_{t}^{\theta} & =\left(-\frac{2 V_{t}^{r} V_{t}^{\theta}}{R_{t}}-\gamma V_{t}^{\theta}\right) d t+\frac{D}{R_{t}} d W_{t}^{\prime 2} \\
d V_{t}^{r} & =\left(R_{t}\left(V_{t}^{\theta}\right)^{2}-\gamma V_{t}^{r}+f\left(R_{t}\right)\right) d t+D d W_{t}^{\prime 1},
\end{aligned}
$$

where the solution $\Theta_{t}$ can be reconstructed from $\left(R_{t}, V_{t}^{r}, V_{t}^{\theta}\right)$.

Remark 5.2 For $\gamma=0$ equation (24) is a Lagrangian SDE with action functional

$$
S=\int_{0}^{t}\left(\frac{1}{2}\left(\left(V_{s}^{x}\right)^{2}+\left(V_{s}^{z}\right)^{2}\right)-F\left(\sqrt{X_{s}^{2}+Z_{s}^{2}}\right)\right) d s+\int_{0}^{t} X_{s} d W_{s}^{1}+\int_{0}^{t} Z_{t} d W_{s}^{2},
$$

where $F(r)=\int_{0}^{r} f(\rho) d \rho$. The flow of the quasi-strong symmetry $V$ leaves the functional $S$ invariant, but equation 24) does not admit a conservation law associated with $V$. Furthermore, as already noted in [45], the reduction of (24] along $V$ allows us to reduce by one (and not by two as in the deterministic case) the dimension of the system.

\subsection{A financial mathematics application: the SABR model}

In this section we discuss a stochastic volatility model used in mathematical finance to describe the stock price $s$ with volatility $u$ under an equivalent martingale measure for $s$ (see [22]). The deep geometric properties of this model, 
related with Brownian motion on the Poincarè plane, are well known and suitably exploited in order to obtain asymptotic expansion formula for options evaluation (see [21]).

The SABR model is a two-dimensional system of the form

$$
\left(\begin{array}{c}
d S_{t} \\
d U_{t}
\end{array}\right)=\left(\begin{array}{cc}
U_{t}\left(S_{t}\right)^{\beta} & 0 \\
\alpha U_{t} \rho & \alpha U_{t} \sqrt{1-\rho^{2}}
\end{array}\right) \cdot\left(\begin{array}{c}
d W_{t}^{1} \\
d W_{t}^{2}
\end{array}\right),
$$

where $\beta, \alpha, \rho \in \mathbb{R}$ and $0<\beta<1$ and $0 \leq \rho \leq 1$.

This SDE admits two symmetries

$$
\begin{aligned}
& V_{1}=\left(Y_{1}, C_{1}, \tau_{1}\right)=\left(\left(\begin{array}{c}
0 \\
1
\end{array}\right), 0,-\frac{2}{u}\right), \\
& V_{2}=\left(Y_{2}, C_{2}, \tau_{2}\right)=\left(\left(\begin{array}{c}
s \\
(1-\beta) u
\end{array}\right), 0,0\right)
\end{aligned}
$$

and we can find a suitable random time change transforming (25) into an integrable SDE as a part of a stochastic transformation $T=(\Phi, B, \eta)$ with $B=I_{2}$ satisfying

$$
\begin{aligned}
Y_{1}(\Phi) & =\left(\begin{array}{l}
0 \\
1
\end{array}\right) \\
Y_{2}(\Phi) & =\left(\begin{array}{c}
1 \\
f(s, u)
\end{array}\right) \\
Y_{1}(\eta) & =-\tau_{1} \eta \\
Y_{2}(\eta) & =0
\end{aligned}
$$

(where $f$ is an arbitrary function). A solution to this system is

$$
\begin{aligned}
\Phi(s, u) & =\left(\begin{array}{c}
\log s \\
u
\end{array}\right), \\
\eta & =\frac{u^{2}}{s^{2-2 \beta}}
\end{aligned}
$$

and putting $t^{\prime}=\int_{0}^{t} \eta\left(S_{s}, U_{s}\right) d s$ and $\left(s^{\prime}, u^{\prime}\right)=\Phi(s, u)$ we obtain the following SDE in the new coordinates

$$
\left(\begin{array}{c}
d S_{t^{\prime}}^{\prime} \\
d U_{t^{\prime}}^{\prime}
\end{array}\right)=\left(\begin{array}{c}
-\frac{1}{2} \\
0
\end{array}\right) d t^{\prime}+\left(\begin{array}{cc}
1 & 0 \\
\alpha e^{(1-\beta) S_{t^{\prime}}^{\prime} \rho} & \alpha e^{(1-\beta) S_{t^{\prime}}^{\prime}} \sqrt{1-\rho^{2}}
\end{array}\right)\left(\begin{array}{c}
d W_{t^{\prime}}^{\prime 1} \\
d W_{t^{\prime}}^{\prime 2}
\end{array}\right)
$$

that is easily integrable.

In [1] the Authors, discussing the non-correlated SABR model $(\rho=0)$, propose a different random time change

$$
\tilde{t}=\int_{0}^{t} U_{s}^{2} d s
$$

in order to derive an analytic formula for the solutions to (25). According to this new time variable, the equation becomes

$$
\left(\begin{array}{c}
d S_{\tilde{t}} \\
d U_{\tilde{t}}
\end{array}\right)=\left(\begin{array}{cc}
\left(S_{\tilde{t}}\right)^{\beta} & 0 \\
\alpha \rho & \alpha \sqrt{1-\rho^{2}}
\end{array}\right)\left(\begin{array}{c}
d \tilde{W}_{\tilde{t}}^{1} \\
d \tilde{W}_{\tilde{t}}^{2}
\end{array}\right)
$$


and its symmetries are

$$
\begin{aligned}
& \tilde{V}_{1}=\left(\left(\begin{array}{l}
0 \\
1
\end{array}\right), 0,0\right), \\
& \tilde{V}_{2}=\left(\left(\begin{array}{c}
s \\
(1-\beta) u
\end{array}\right), 0,2(1-\beta)\right) .
\end{aligned}
$$

Therefore the time change $\tilde{t}$ transforms $V_{1}$ into the strong symmetry $\tilde{V}_{1}$ and $V_{2}$ into the symmetry $\tilde{V}_{2}$, which, since $\beta$ is a constant, corresponds to a deterministic time change. The symmetry $\tilde{V}_{2}$, restricted to the $s$ variable, is the symmetry of a Bessel process: indeed the process $S$ solves an equation for a spatial changed Bessel process. The Bessel process is one of the few one-dimensional stochastic processes whose transition probability is explicitly known and is a special case of the general affine processes class ( see 15]). We remark that the time change $\tilde{t}$ can be uniquely characterized by the special form of $\tilde{V}_{1}$ and $\tilde{V}_{2}$, whose expression can be recovered within our symmetry analysis. Finally this last example suggests the possibility of extending the integrability notion to processes which are not progressively reconstructible from gaussian processes but, more in general, from other processes with notable analytical properties, such as Bessel process, affine processes or other processes.

\section{Acknowledgements}

This work was supported by National Group of Mathematical Physics (GNFMINdAM).

\section{References}

[1] Alexandre Antonov and Michael Spector. Advanced analytics for the sabr model. Available at SSRN 2026350, 2012.

[2] Alexis Arnaudon, Alex L. Castro, and Darryl D. Holm. Noise and dissipation on coadjoint orbits. arXiv preprint arXiv:1601.02249, 2016.

[3] Marc Arnaudon, Xin Chen, and Ana Bela Cruzeiro. Stochastic EulerPoincaré reduction. J. Math. Phys., 55(8):081507, 17, 2014.

[4] Florin Avram, Nikolai N. Leonenko, and Nenad Šuvak. On spectral analysis of heavy-tailed Kolmogorov-Pearson diffusions. Markov Process. Related Fields, 19(2):249-298, 2013.

[5] Mátyás Barczy, Leif Döring, Zenghu Li, and Gyula Pap. On parameter estimation for critical affine processes. Electron. J. Stat., 7:647-696, 2013.

[6] Denis Belomestny and Markus Reiß. Spectral calibration of exponential Lévy models. Finance Stoch., 10(4):449-474, 2006.

[7] Jean-Michel Bismut. Mécanique aléatoire, volume 866 of Lecture Notes in Mathematics. Springer-Verlag, Berlin-New York, 1981.

[8] Lisa Borland. A theory of non-Gaussian option pricing. Quant. Finance, 2(6):415-431, 2002.

[9] Giacomo Bormetti and Sofia Cazzaniga. Multiplicative noise, fast convolution and pricing. Quant. Finance, 14(3):481-494, 2014. 
[10] Francesco Cordoni and Luca Di Persio. Lie symmetry approach to the cev model. International Journal of Differential Equations and Applications, $13(3), 2014$.

[11] Mark Craddock. Fourier type transforms on Lie symmetry groups. J. Math. Phys., 56(9):091501, 28, 2015.

[12] Mark Craddock and Kelly A. Lennox. Lie symmetry methods for multidimensional parabolic PDEs and diffusions. J. Differential Equations, 252(1):56-90, 2012.

[13] Christa Cuchiero, Martin Keller-Ressel, and Josef Teichmann. Polynomial processes and their applications to mathematical finance. Finance Stoch., 16(4):711-740, 2012.

[14] Francesco C. De Vecchi, Paola Morando, and Stefania Ugolini. Symmetries of stochastic differential equations: A geometric approach. Journal of Mathematical Physics, 57(6), 2016.

[15] Darrell Duffie, Damir Filipović, and Walter Schachermayer. Affine processes and applications in finance. Ann. Appl. Probab., 13(3):984-1053, 2003.

[16] Julie L. Forman and Michael Sørensen. The Pearson diffusions: a class of statistically tractable diffusion processes. Scand. J. Statist., 35(3):438-465, 2008 .

[17] Rudolf Friedrich and Joachim Peinke. Description of a turbulent cascade by a fokker-planck equation. Physical Review Letters, 78(5):863, 1997.

[18] Giuseppe Gaeta and Niurka Rodríguez Quintero. Lie-point symmetries and stochastic differential equations. J. Phys. A, 32(48):8485-8505, 1999.

[19] Thomas C. Gard. Introduction to stochastic differential equations, volume 114 of Monographs and Textbooks in Pure and Applied Mathematics. Marcel Dekker, Inc., New York, 1988.

[20] Fatemeh Ghasemi, Muhammad Sahimi, Joachim Peinke, and M. Reza Rahimi Tabar. Analysis of non-stationary data for heartrate fluctuations in terms of drift and diffusion coefficients. Journal of biological physics, 32(2):117-128, 2006.

[21] Patrick Hagan, Andrew Lesniewski, and Diana Woodward. Probability distribution in the SABR model of stochastic volatility. In Large deviations and asymptotic methods in finance, volume 110 of Springer Proc. Math. Stat., pages 1-35. Springer, Cham, 2015.

[22] Patrick S. Hagan, Deep Kumar, Andrew S. Lesniewski, and Diana E. Woodward. Managing smile risk. The Best of Wilmott, page 249, 2002.

[23] Steven L. Heston. A closed-form solution for options with stochastic volatility with applications to bond and currency options. Review of financial studies, 6(2):327-343, 1993.

[24] Simon Hochgerner and Tudor S. Ratiu. Geometry of non-holonomic diffusion. J. Eur. Math. Soc. (JEMS), 17(2):273-319, 2015. 
[25] Darryl D. Holm. Variational principles for stochastic fluid dynamics. In Proceedings of the Royal Society of London A: Mathematical, Physical and Engineering Sciences, volume 471, page 20140963. The Royal Society, 2015.

[26] Roman Kozlov. Symmetries of systems of stochastic differential equations with diffusion matrices of full rank. J. Phys. A, 43(24):245201, 16, 2010.

[27] Joan-Andreu Lázaro-Camí and Juan-Pablo Ortega. Stochastic Hamiltonian dynamical systems. Rep. Math. Phys., 61(1):65-122, 2008.

[28] Joan-Andreu Lázaro-Camí and Juan-Pablo Ortega. Reduction, reconstruction, and skew-product decomposition of symmetric stochastic differential equations. Stoch. Dyn., 9(1):1-46, 2009.

[29] Joan-Andreu Lázaro-Camí and Juan-Pablo Ortega. Superposition rules and stochastic Lie-Scheffers systems. Ann. Inst. Henri Poincaré Probab. Stat., 45(4):910-931, 2009.

[30] Paul Lescot and Jean-Claude Zambrini. Probabilistic deformation of contact geometry, diffusion processes and their quadratures. In Seminar on Stochastic Analysis, Random Fields and Applications $V$, volume 59 of Progr. Probab., pages 203-226. Birkhäuser, Basel, 2008.

[31] Xue-Mei Li. An averaging principle for a completely integrable stochastic Hamiltonian system. Nonlinearity, 21(4):803-822, 2008.

[32] Matthew Lorig, Stefano Pagliarani, and Andrea Pascucci. A family of density expansions for Lévy-type processes. Ann. Appl. Probab., 25(1):235$267,2015$.

[33] Simon J. A. Malham and Anke Wiese. Stochastic Lie group integrators. SIAM J. Sci. Comput., 30(2):597-617, 2008.

[34] Umberto Marini Bettolo Marconi, Andrea Puglisi, Lamberto Rondoni, and Angelo Vulpiani. Fluctuation-dissipation: response theory in statistical physics. Physics reports, 461(4):111-195, 2008.

[35] Sergey V. Meleshko, Yurii N. Grigoriev, Nail K. Ibragimov, and Vladimir F. Kovalev. Symmetries of integro-differential equations: with applications in mechanics and plasma physics, volume 806. Springer Science \& Business Media, 2010.

[36] Ieke Moerdijk and Janez Mrčun. Introduction to foliations and Lie groupoids, volume 91 of Cambridge Studies in Advanced Mathematics. Cambridge University Press, Cambridge, 2003.

[37] Peter J. Olver. Applications of Lie groups to differential equations, volume 107 of Graduate Texts in Mathematics. Springer-Verlag, New York, second edition, 1993.

[38] Eckhard Platen and Nicola Bruti-Liberati. Numerical solution of stochastic differential equations with jumps in finance, volume 64 of Stochastic Modelling and Applied Probability. Springer-Verlag, Berlin, 2010.

[39] Nicolas Privault and Jean-Claude Zambrini. Stochastic deformation of integrable dynamical systems and random time symmetry. J. Math. Phys., 51(8):082104, 19, 2010. 
[40] Udo Seifert. Stochastic thermodynamics, fluctuation theorems and molecular machines. Reports on Progress in Physics, 75(12):126001, 2012.

[41] William T. Shaw and Marcus Schofield. A model of returns for the postcredit-crunch reality: hybrid brownian motion with price feedback. Quantitative Finance, 15(6):975-998, 2015.

[42] Hans Stephani. Differential equations: their solution using symmetries. Cambridge University Press, Cambridge, 1989.

[43] È. B. Vinberg. Lie groups and Lie algebras, III, volume 41 of Encyclopaedia of Mathematical Sciences. Springer-Verlag, Berlin, 1994.

[44] Grzegorz Wilk and Zbigniew Włodarczyk. Interpretation of the nonextensivity parameter $\mathrm{q}$ in some applications of tsallis statistics and Lévy distributions. Physical Review Letters, 84(13):2770, 2000.

[45] Nguyen T. Zung and Nguyen T. Thien. Reduction and integrability of stochastic dynamical systems. arXiv preprint arXiv:1410.5492, 2014. 